\title{
Numerical Study of the Effects of Shear Deformation and Superimposed Hydrostatic Pressure on the Formability of AZ31B Sheet at Room Temperature
}

\author{
H. Wang ${ }^{1,2^{*}}$, P.D. Wu ${ }^{1}$, S.Y. Lee ${ }^{3}$ J. Wang ${ }^{2}$, K.W. Neale ${ }^{4}$ \\ ${ }^{1}$ Department of Mechanical Engineering, McMaster University, \\ Hamilton, Ontario L8S 4L7, Canada \\ ${ }^{2}$ Materials Science and Technology Division, Los Alamos National Laboratory, \\ Los Alamos, NM 87544, USA \\ ${ }^{3}$ Department of Materials Science and Engineering, Chungnam National University, \\ Daejeon 305-764, South Korea \\ ${ }^{4}$ Faculty of Engineering, University of Sherbrooke, \\ Sherbrooke, Quebec J1K 2R1, Canada
}

\begin{abstract}
The effect of the shear deformation and the superimposed hydrostatic pressure on the formability of magnesium alloy sheet is simulated in terms of the forming limit diagram (FLD). The model employed is the elastic viscoplastic selfconsistent (EVPSC) crystal plasticity model, which accounts for both slip and twinning systems as the deformation mechanisms. The conventional sheets have low formability at room temperature due to the strong basal texture developed by the rolling process. However differential speed rolling process develops relatively weak basal texture by introducing shear deformation. Therefore the formability of the sheets produced by differential speed rolling is enhanced. In terms of the superimposed hydrostatic pressure, it delays the onset of necking and therefore improves the formability of sheets. In addition, the effect of crystal elasticity on the formability of sheets is numerically studied.
\end{abstract}

Keywords: Crystal Plasticity; Magnesium; Formability; Differential Speed Rolling; Superimposed pressure.

* Corresponding authors. Tel.: +1-505-664-0321, E-mail address: wanghm@lanl.gov (Huamiao Wang). 


\section{Introduction}

Magnesium alloys are of great interest due to their light weight to reduce the energy consumption. However, poor formability of the conventionally rolled sheets at room temperature limits their applications. Among plastic deformation mechanisms, Basal, Prismatic and Pyramidal slips and Extension twinning, the rolled magnesium alloy sheets do not favor Prismatic and Pyramidal slips under in-plane deformation because of their high resistances and the strong basal textures developed by the conventional rolling process, Wang et al. (2010c; 2011) found that artificially weakening the basal texture can improve the formability according to their modeling of the forming limit diagram (FLD) using crystal plasticity model.

In reality, weakening the basal texture has been achieved by introducing shear deformation during rolling process. Consequently, the associated ductility under uniaxial deformation has been improved (Mukai et al., 2001; Agnew et al., 2004; Chino et al., 2008, Huang et al., 2008). Among these processes, the differential speed rolling (DSR) (Huang et al., 2008) is possible to meet the commercial need with high productivity and low cost. Therefore one part of present study is focusing on studying the effect of DSR induced shear deformation on the formability of AZ31B sheet using the elastic viscoplastic self-consistent (EVPSC) model (Wang et al., 2010d). Another part of present study is focusing on studying the influence of superimposed pressure on the formability of magnesium alloy sheet. The pressure influence on other materials has been intensively studied (Bridgman, 1952; French et al., 1973; French and Weinrich, 1975; Weinrich and French, 1976; Brownrigg et al., 1983; Korbel et al., 1984; Spitzig and Richmond, 1984; Ashby et al., 1985; Kao et al., 1989; 1990; Liu and Lewandowski, 1993; Lewandowski and Lowhaphandu, 1998; Gimple et al., 2001; Wu et al, 2009). The key finding is that the hydrostatic pressure postpones or completely removes the development of micro-voids 
or micro-cracks in the material and thus delays failure of the materials. In order to understand the effect of hydrostatic pressure on the formability of AZ31B sheets, we simulated the proportional in-plane straining with simultaneously superimposed hydrostatic pressure. The effect of hydrostatic pressure on the forming limit strains at various strain paths is studied. In addition, the effect of crystal elasticity of magnesium alloy on the formability of sheets is numerically studied. As reported by Wu et al. (1997; 1998), elastic constants have influence on the prediction of FLDs for materials with the face centered cubic (FCC) crystal structure. It is expected that the crystal elasticity also has an influence on the prediction of the FLDs of the magnesium alloy sheets.

\section{Model and approach description}

\subsection{EVPSC model}

The EVPSC model is only briefly described here. The development, validation, evaluation and applications of the EVPSC model can be found elsewhere (Wang et al., 2010a, b, c, d, e; 2012a, d; Wu et al., 2012; 2013a, b, c; Lee et al., 2014; Wang et al., 2015). The constitutive equation for a crystal is:

$$
\stackrel{\nabla}{*}^{*}=\boldsymbol{L}: \dot{\boldsymbol{\varepsilon}}^{e}-\boldsymbol{\sigma} \operatorname{tr}\left(\dot{\boldsymbol{\varepsilon}}^{e}\right)
$$

where $\nabla^{*}$ is the Jaumann rate of the Cauchy stress $\boldsymbol{\sigma}$ based on the lattice spin tensor $\boldsymbol{w}^{e}, \boldsymbol{L}$ is the elastic stiffness tensor and $\dot{\boldsymbol{\varepsilon}}^{e}$ is the elastic strain rate tensor. $\boldsymbol{L}$ is a function of the crystal elastic constants $C_{i j}$ (Wang and Mora, 2008).

Plastic deformation of a crystal is attributed to crystallographic slip and twinning on system $\left(\boldsymbol{s}^{\alpha}, \boldsymbol{n}^{\alpha}\right)$. Here, $\boldsymbol{s}^{\alpha}$ and $\boldsymbol{n}^{\alpha}$ are the slip/twinning direction and the direction normal to the 
slip/twinning plane for system $\alpha$, respectively. The grain (crystal) level plastic strain rate $\dot{\boldsymbol{\varepsilon}}^{p}$ is given by (Asaro and Needleman, 1985):

$$
\dot{\boldsymbol{\varepsilon}}^{p}=\dot{\gamma}_{0} \sum_{\alpha} \boldsymbol{P}^{\alpha}\left|\tau^{\alpha} / \tau_{c r}^{\alpha}\right|^{\frac{1}{m}} \operatorname{sgn}\left(\tau^{\alpha}\right)
$$

where $\dot{\gamma}_{0}$ is a reference value for the slip/twinning rate, $m$ is the slip/twinning rate sensitivity, $\boldsymbol{P}^{\alpha}=\left(\boldsymbol{s}^{\alpha} \boldsymbol{n}^{\alpha}+\boldsymbol{n}^{\alpha} \boldsymbol{s}^{\alpha}\right) / 2$ is the Schmid tensor for system $\alpha$, and $\tau^{\alpha}=\boldsymbol{\sigma}: \boldsymbol{P}^{\alpha}$ and $\tau_{c r}^{\alpha}$ are the resolved shear stress and critical resolved shear stress (CRSS) for system $\alpha$, respectively. The evolution of $\tau_{c r}^{\alpha}$ due to hardening processes is given by:

$$
\dot{\tau}_{c r}^{\alpha}=\frac{\mathrm{d} \hat{\tau}^{\alpha}}{\mathrm{d} \gamma_{a c}} \sum_{\beta} h^{\alpha \beta} \dot{\gamma}^{\beta}
$$

where $\gamma_{a c}=\sum_{\alpha} \int\left|\dot{\gamma}^{\alpha}\right| d t$ is the accumulated shear strain in the grain, and $h^{\alpha \beta}$ are the latent hardening coupling coefficients, which empirically accounts for the obstacles on system $\alpha$ associated with system $\beta . \hat{\tau}^{\alpha}$ is the threshold stress and characterized by:

$$
\hat{\tau}^{\alpha}=\tau_{0}^{\alpha}+\left(\tau_{1}^{\alpha}+h_{1}^{\alpha} \gamma_{a c}\right)\left(1-\exp \left(-h_{0}^{\alpha} \gamma_{a c} / \tau_{1}^{\alpha}\right)\right)
$$

Here, $\tau_{0}, h_{0}, h_{1}$ and $\tau_{0}+\tau_{1}$ are the initial CRSS, the initial hardening rate, the asymptotic hardening rate, and the back-extrapolated CRSS, respectively. The Predominant Twin Reorientation (PTR) twinning model (Tomé et al., 1991) is employed to model twinning. PTR model prevents grain reorientation by twinning until the twin volume fraction reaches a threshold value $V^{\text {th }}=A^{1}+A^{2} V_{\text {eff }} / V_{a c c}$, where $A^{1}$ and $A^{2}$ are two constants, $V_{a c c}$ and $V_{\text {eff }}$ are the weighted volume fraction of twinned region and the volume fraction of twin terminated grains, respectively. 
The self-consistent model treats each grain as an ellipsoidal inclusion embedded in a Homogeneous Effective Medium (HEM), which is an aggregate of all the grains. Interactions between a grain and the HEM are described through the Eshelby inclusion formalism (Eshelby, 1957). The self-consistency criteria and the single crystal constitutive rule are solved simultaneously in an increment, which ensures the consistency between the grain-level stresses and strains and the ones imposed on the HEM. The behavior of the inclusion (single crystal) and the HEM can be linearized as follows (Wang et al., 2010d):

$$
\begin{gathered}
\dot{\boldsymbol{\varepsilon}}=\dot{\boldsymbol{\varepsilon}}^{e}+\dot{\boldsymbol{\varepsilon}}^{p}=\boldsymbol{M}^{e}: \dot{\boldsymbol{\sigma}}+\boldsymbol{M}^{p}: \boldsymbol{\sigma}+\dot{\boldsymbol{\varepsilon}}^{0} \\
\dot{\overline{\boldsymbol{\varepsilon}}}=\overline{\boldsymbol{M}}^{e}: \dot{\overline{\boldsymbol{\sigma}}}+\overline{\boldsymbol{M}}^{p}: \overline{\boldsymbol{\sigma}}+\dot{\overline{\boldsymbol{\varepsilon}}}^{0}
\end{gathered}
$$

where $\dot{\boldsymbol{\varepsilon}}, \dot{\boldsymbol{\sigma}}, \boldsymbol{M}^{e}, \boldsymbol{M}^{p}$, and $\dot{\boldsymbol{\varepsilon}}^{0}$ are the strain rate, stress rate, elastic compliance, the viscoplastic compliance, and the back-extrapolated term for the grain, respectively. $\dot{\overline{\boldsymbol{\varepsilon}}}, \dot{\overline{\boldsymbol{\sigma}}}, \overline{\boldsymbol{M}}^{e}, \overline{\boldsymbol{M}}^{p}$, and $\dot{\overline{\boldsymbol{\varepsilon}}}^{0}$ are the corresponding terms for the HEM. The grain-level stresses and strain rates are related self-consistently to the corresponding values for the HEM as follows:

$$
\dot{\boldsymbol{\varepsilon}}-\dot{\overline{\boldsymbol{\varepsilon}}}=-\tilde{\boldsymbol{M}}^{e}:(\dot{\boldsymbol{\sigma}}-\dot{\overline{\boldsymbol{\sigma}}})-\tilde{\boldsymbol{M}}^{p}:(\boldsymbol{\sigma}-\overline{\boldsymbol{\sigma}})
$$

where the interaction tensors $\tilde{\boldsymbol{M}}^{e}$ and $\tilde{\boldsymbol{M}}^{p}$ are given by:

$$
\tilde{\boldsymbol{M}}^{e}=\left(\boldsymbol{I}-\boldsymbol{S}^{e}\right)^{-1}: \boldsymbol{S}^{e}: \overline{\boldsymbol{M}}^{e}, \tilde{\boldsymbol{M}}^{p}=\left(\boldsymbol{I}-\boldsymbol{S}^{p}\right)^{-1}: \boldsymbol{S}^{p}: \overline{\boldsymbol{M}}^{p}
$$

Here, $\boldsymbol{S}^{e}$ and $\boldsymbol{S}^{e}$ are the elastic and the viscoplastic Eshelby tensors for a given grain, respectively. $\boldsymbol{I}$ is the identity tensor. Several self-consistent schemes (SCSs) have been proposed for the linearization of Eq. (5). Affine self-consistent scheme was found to give the best overall performance (Wang et al., 2010a; b; e) and is employed in the present study.

\subsection{M-K approach}


The concept of the FLD has been developed to indicate the onset of sheet necking (see, e.g. Hecker, 1975). It is now a standard tool for characterizing materials in terms of their overall forming behavior. The M-K approach is widely employed to construct the FLDs theoretically or numerically (Marciniak and Kuczynski, 1967). The combination of the self-consistent models and the M-K approach to construct the FLDs has been successfully applied to study the polycrystalline materials (Neil and Agnew, 2009; Wang et al., 2011). In present study we employ the EVPSC model with the Affine scheme and the M-K approach. The detailed approach for constructing the FLDs is referred to Wang et al. (2011).

The $\mathrm{M}-\mathrm{K}$ approach assumes the existence of material imperfections in the form of grooves that is initially inclined at an angle $\psi_{I}$ with respect to the $x_{1}$ reference direction (Fig. 1). A minor intrinsic imperfection in load bearing capacity throughout a deforming sheet can lead to unstable growth of strain in the region of the imperfection, and subsequently cause localized necking and failure. The intrinsic geometric non-uniformity $f$ is defined as the ratio between the thicknesses inside and outside the grooves and only quantitatively influences the constructed FLDs (Wu et al., 1997). The initial value of $f$ is set to be 0.99 in the present study.

The influence of the superimposed hydrostatic pressure on formability is studied by considering a non-proportional deformation history using combination of two liner deformation paths. $\sigma_{i j}$ and $\varepsilon_{i j}$ (instead of $\bar{\sigma}_{i j}$ and $\bar{\varepsilon}_{i j}$ ) are used to denote the macroscopic stresses and strains, respectively. The first loading path is to apply desired pressure $p$ incrementally on material both outside and inside the imperfection

$$
\dot{\sigma}_{11}=\dot{\sigma}_{22}=\dot{\sigma}_{33}=-\dot{p}
$$


The deformation inside and outside the groove remains very small during this nearly elastic loading even when $p$ is very high. When the designed hydrostatic pressure $p$ is reached, the current non-uniformity $f$ and deformation state inside and outside the groove are used as the starting state for the subsequent stain path in the FLD analysis. Various strain paths should be investigated to construct an entire FLD, therefore in the subsequent linear strain path, the deformation outside the groove is prescribed to be:

$$
\frac{\dot{\varepsilon}_{22}}{\dot{\varepsilon}_{11}}=\rho, \dot{\varepsilon}_{12}=0, W_{12}=0
$$

where $\dot{\varepsilon}_{11}$ and $\dot{\varepsilon}_{22}$ are the (principal) logarithmic strain rates and the $W_{i j}$ values are components of the spin tensor. It is further assumed that $\dot{\varepsilon}_{13}=\dot{\varepsilon}_{23}=W_{13}=W_{23}=0$, while $\dot{\varepsilon}_{33}$ is specified by the condition $\dot{\sigma}_{33}=0$ ( or $\sigma_{33} \equiv p$ ). The entire FLD can be constructed by changing the ratio $\rho$ between the strain rates $\dot{\varepsilon}_{22}$ and $\dot{\varepsilon}_{11}$.

Under the imposed deformations described in equation (10), the evolution of the groove orientation $\psi$ is given by

$$
\dot{\psi}=n_{1} n_{2}\left(\dot{\varepsilon}_{11}-\dot{\varepsilon}_{22}\right)-\left(n_{1}^{2}-n_{2}^{2}\right) \dot{\varepsilon}_{12}
$$

where $n_{1}=\cos \psi$ and $n_{2}=\sin \psi$ are the components of the unit normal to the band (Fig. 1). The compatibility and equilibrium conditions at the groove interface are given respectively as follows:

$$
\begin{gathered}
\dot{\varepsilon}_{\alpha \beta}^{b}=\dot{\varepsilon}_{\alpha \beta}+\frac{1}{2}\left(v_{\alpha} n_{\beta}+v_{\alpha} n_{\beta}\right), w_{\alpha \beta}^{b}=w_{\alpha \beta}+\frac{1}{2}\left(v_{\alpha} n_{\beta}-v_{\alpha} n_{\beta}\right) \\
f n_{\alpha} \sigma_{\alpha \beta}^{b}=n_{\alpha} \sigma_{\alpha \beta}
\end{gathered}
$$


The Greek indices range from 1 to $2 . v_{\alpha}(\alpha=1,2)$ and $\dot{\varepsilon}_{33}^{b}$ are the unknown variables that are determined from these two equations and the condition $\dot{\sigma}_{33}^{b}=0$. The intrinsic geometric nonuniformity $f$ is updated based on the relation

$$
\dot{f}=f\left(\dot{\varepsilon}_{33}^{b}-\dot{\varepsilon}_{33}\right)
$$

Therefore deformation and stress state inside the groove are completely determined. The onset of sheet necking is defined by the occurrence of a much higher maximum principal logarithmic strain rate inside the band than outside, i.e., $\dot{\varepsilon}^{b} / \dot{\varepsilon}_{11} \geq R$, where $\dot{\varepsilon}^{b}$ and $R$ represent the maximum strain rate inside the band and the threshold value for the onset of necking, respectively. The threshold value $R$, which was chosen from $10^{2}$ to $10^{5}$, only affects the FLDs quantitatively (Wu et al., 1997; Neil and Agnew, 2009; Wang et al., 2011). Here $R=10^{4}$ is employed in present study. The limit strains are the corresponding principal logarithmic strains $\varepsilon_{11}^{*}$ and $\varepsilon_{22}^{*}$ outside the band. For a real sheet, numerous initial imperfections can exist with different orientations. A conservative estimate of the forming limit strain is the minimum limit strain obtained with different initial band orientation $\psi_{I}$ at a strain path. The entire FLD of a sheet is constructed by repeating the procedure for different strain paths with $\rho$ ranges from -0.5 to 1 .

\section{Results and discussion}

We studied magnesium alloy AZ31B sheet in this work. Texture and mechanical properties of the material is the same as that described by Jain and Agnew (2007). The initial crystallographic texture of the sheet consists of 2160 grains with independent orientations and weights. The $\{0001\}$ and $\{10 \overline{1} 0\}$ pole figures of the texture $(\gamma=0$ in Fig. 2$)$ show that the 
grains tend to have their basal planes oriented perpendicular (c-axis parallel) to the normal direction (ND) of the sheet. The plastic deformation modes of AZ31B are assumed to be slip in Basal $\langle a\rangle(\{0001\}<11 \overline{2} 0>)$, Prismatic $\langle a\rangle \quad(\{10 \overline{1} 0\}<11 \overline{2} 0>)$ and Pyramidal $\langle c+a\rangle$ $(\{\overline{1} \overline{1} 22\}<\overline{1} \overline{1} 23>$ ) slip systems, and twinning on the $\{10 \overline{1} 2\}<\overline{1} 011>$ tensile twin system (Wang et al. 2010a).

It was reported that magnesium alloys showed a rate dependence even at low strain rate (e.g. Kurukuri et al., 2014). The reference slip/twinning rate and the rate sensitivity are prescribed to be the same for all slip/twinning systems: $\dot{\gamma}_{0}=0.001 \mathrm{~s}^{-1}$ and $m=0.05$, respectively. The room temperature elastic constants of magnesium single crystal are $C_{11}=58.0, C_{12}=25.0$, $C_{13}=20.8, C_{33}=61.2$ and $C_{44}=16.6$ (unit of GPa) (Simmons and Wang, 1971). The set of CRSSs and hardening parameters for each mode are estimated by fitting numerical simulations of uniaxial tension and compression along the RD to the corresponding experimental flow curves. In these simulations, a strain rate, $\dot{\varepsilon}_{11}=0.001 \mathrm{~s}^{-1}$, is prescribed in the loading direction, and the macroscopic stress components are enforced to be zero, except for the stress $\sigma_{11}$ along the loading direction. The material parameters associated with slip systems are determined from uniaxial tension along the $\mathrm{RD}$, while values for the material parameters associated with twinning are determined from uniaxial compression along the RD. Fig. 3 presents the uniaxial tension and compression true stress and plastic strain curves along the RD. the importance of twinning in compression is clearly revealed by the characteristic S-shape of the flow curve. The EVPSC model associated with Affine self-consistent scheme fits the experimental stress strain curves well. Table 1 contains the material parameters obtained from the simulations. These parameters are used in all subsequent simulations. 


\subsection{Influence of elastic constants on FLDs}

Magnesium alloys in numerically simulations was referred as either the anisotropic elastic constants of $C_{i j}$ with five independent components (Simmons and Wang, 1971; Wang and Mora, 2008; Wang et al., 2012a) or isotropic elastic constants of Young's modulus $E \approx 45 \mathrm{GPa}$ and Poisson ratio $v \approx 0.3$ (Suzuki et al., 2011; Gupta and Sharon, 2011). When elastic deformation was not the interest, high values of the elastic constants were used to eliminate the elastic deformations (Wang et al. 2010d; 2011). The effect of the elastic constants on the FLDs of magnesium alloy sheets has never been investigated and remains unknown. For rolled aluminum alloy sheets, people have noticed that there is a dip near in-plane plane strain tension in the measured FLDs (see e.g. Ratchev et al., 1994; Wu et al., 1998). However, this experimentally observed feature had not been numerically re-produced until Wu et al. (1997), who demonstrated that it is the elastic effect which determines the shape of the FLD near inplane plane strain tension. It is apparently impossible for an FLD analysis neglecting elasticity to reproduce the dip observed in the experimental determined FLDs. Wu et al. (1997) also indicated that increasing the elastic modulus of a sheet metal improves its formability.

Therefore it is worthy to investigate the influence of elasticity on FLDs of magnesium alloy sheet. We considered four sets of elastic constants: 1) the anisotropic elastic constants $C_{i j}$; 2) the isotropic elastic constants $E=45 G P a$ and $v=0.3$; 3) 'rigid'-plastic material with anisotropic elastic constants of $20 \times C_{i j}$; and 4) 'rigid'-plastic material with isotropic elastic constants of $20 \times E=900 G P a$ and $v=0.3$. The term 'rigid' plastic is used here because the elastic deformation is negligible and the stress-strain curve of this material is almost identical to that of the corresponding ideal rigid-plastic material. The predicted FLDs are presented in Fig. 4. 
The legends $C_{i j}, E=45 \mathrm{GPa}, 20 C_{i j}$ and $E=900 \mathrm{GPa}$ represent the FLDs for different anisotropic single crystal elastic constants, respectively. It is clear that the difference in predicted FLDs is small for the strain paths $-0.5 \leq \rho \leq 0.1$. However, this difference becomes significant for the strain paths $\rho>0.1$. It is observed that the predicted FLD for anisotropic crystal elastic constants $C_{i j}$ is higher than that for isotropic crystal elastic constant $E=45 \mathrm{GPa}$. It is interesting to note that the predicted FLDs for 'rigid'-plastic materials ( $20 C_{i j}$ and $E=900 \mathrm{GPa}$ ) are almost the same, which indicates that the constitutive behaviors are identical for these two cases where the elastic deformation is negligible. Since plastic deformation is the dominant deformation involved for constructing the FLDs, the overall shapes of all FLDs studied are similar to each other. The effect of elasticity on the FLD shown in Fig.4 for Mg alloy sheet in the present paper is similar to the one for Al alloy sheet revealed by Wu et al. (1997). However, it is not clear how elasticity affects plastic deformation mechanism and thus influences the FLD.

\subsection{Influence of shear deformation on FLDs}

Differential speed rolling (DSR) is a process carried out at different rotation speeds for upper and lower rolls so that intense shear deformation can be introduced throughout the sheet thickness, which can result in grain refinement and change in texture. DSR can be decomposed into two parts in terms of deformation: shear deformation in the RD - ND (axis 1 - axis 3) plane and conventional rolling along ND (Fig. 5). Huang et al. (2008) reported that the conventional rolling strengthened the basal texture of the magnesium alloy sheets. Therefore shear deformation is the one weakens the basal texture. The effect of the shear deformation reflects the effect of DSR on the formability of magnesium alloy sheets. The boundary conditions for the shear deformation is prescribed to be $\dot{\sigma}_{11}=\dot{\sigma}_{33}=0, \quad \dot{\varepsilon}_{22}=\dot{\varepsilon}_{12}=\dot{\varepsilon}_{23}=0, \quad \dot{\varepsilon}_{13}=\dot{\gamma} / 2 \neq 0$, 
$\dot{\varepsilon}_{12}=\dot{\varepsilon}_{23}=0$. The AZ31B sheet is sheared to various shear strain levels. In these simulations, a strain rate of $\dot{\gamma}=2 \dot{\varepsilon}_{13}=0.001 \mathrm{~s}^{-1}$ is prescribed in the loading direction. The textures at shear levels of $\gamma=0.3,0.6,0.9$ are shown in Fig. 2. The shear deformation not only moves the basal poles away from the normal direction of the sheet, but also strengthens the texture at the preferred orientation of $\sim 15^{\circ}$ away from the ND.

Figure 6 gives the intensity of the basal texture as a function of the shear deformation. The intensity is calculated as the weight fraction of the grains with a maximum orientation difference to the ideal basal orientation less than a cut-off angle (Wang et al., 2010c; 2011). Two cut-off angle, $15^{\circ}$ and $45^{\circ}$, are used to evaluate the intensity of the basal texture. The ideal basal grain corresponds to perfect alignment of the basal pole with the normal direction. For the cut-off angle of $15^{\circ}$, it is noted that the calculated intensity for $\gamma<0.2$ remains constant, then the intensity decreases rapidly when the shear deformation $\gamma$ is greater than 0.2 . For the cut-off angle of $45^{\circ}$, the intensity decreases rapidly with increasing the shear deformation up to $\gamma=0.3$, then increases gradually with further increasing the shear deformation. Therefore in order to study the influence of shear deformation on formability of the magnesium alloys, the textures generated from the shear deformations are used as the initial textures to construct the forming limit diagram of AZ31B sheet. The initial non-uniformity is taken as $f_{0}=0.99$ when constructing the FLDs.

Figure 7 presents the influence of shear deformation on the predicted FLDs. As can be seen, the formability of HCP sheets can be improved through changing the texture by imposing shear deformation $\gamma$. The improvement of formability of AZ31B sheet increases with increasing the shear deformation up to 0.3 and deceases with further increasing the shear deformation. It 
was reported that the higher the intensity of the basal texture, the poor the formability of the magnesium alloy sheets (Huang et al., 2008; Wang et al., 2010c; 2011). Figs. 6 and 7 show that the predicted FLDs can be better explained by the intensity of the basal texture with the cut-off angle of $45^{\circ}$. This is reasonable because the grains with c-axes oriented $45^{\circ}$ from the ND are easy to activate basal slip. Using the cut-off angle of $15^{\circ}$ to calculate the intensity of basal texture can reflect the change in the basal texture if the texture is only rotated (e.g., Wang et al. 2010c; 2011). However in present study, the texture is altered by the shear deformation. Therefore the dependency of formability of magnesium alloy sheets on the intensity of basal texture and furthermore on the shear deformation (or DSR) will be explained by using the cut-off angle of $45^{\circ}$. With the cut-off angle of $45^{\circ}$, the intensity of the basal texture decreases to its minimum at shear deformation of 0.3 and then increases gradually with increasing the shear deformation. The FLD increases to its highest value at shear deformation of 0.3 and then decreases gradually with increasing the shear deformation. Interestingly, the FLDs of shear deformation of 0.2 and 0.6 are very similar, which is because there basal intensities are almost the same. The effect of shear deformation on formability is more even more clearly exhibited in Fig. 8, which shows the predicted major limit strains versus the shear deformation $\gamma$ for strain paths $\rho$ of $-0.5,0$ and 1 . It is clear that the predicted limit strain is dramatically increased when the shear deformation $\gamma \leq 0.3$ and the increase of the limit strain is reduced with further increasing the shear deformation, which is consistent with the basal intensity in terms of the cutoff angle of $45^{\circ}$.

Figure 9 gives the relative activity of the deformation mechanisms outside the groove under in-plane plane tension $(\rho=0)$ with various shear-deformed textures. The basal slip and extension twin activities increase with increasing the shear strain up to $\gamma=0.3$, then decrease 
slightly. Therefore the higher formability of the DSR produced magnesium alloy sheets is attributed to the activation of more basal slip and extension twin through altering the texture by applying shear deformation.

\subsection{Influences of superimposed hydrostatic pressure on FLDs}

The influences of superimposed hydrostatic pressure on sheet metal formability of magnesium sheet are further assessed. The sheet is pre-loaded with the condition $\dot{\sigma}_{11}=\dot{\sigma}_{22}=\dot{\sigma}_{33}=-\dot{p}$ to hydrostatic pressure $p$. When the designed hydrostatic pressure $p$ is reached, the current non-uniformity $f$ and deformation state inside and outside the groove are used as the starting state for the subsequent stain path in the FLD analysis.

Figure 10 presents the stress $\left(\sigma_{11}\right)$ and strain $\left(\varepsilon_{11}\right)$ curves for the material point outside the imperfection band for the strain path of plane strain tension $(\rho=0)$ under various superimposed hydrostatic pressures. The calculations are terminated at necking with $\dot{\varepsilon}^{b} / \dot{\varepsilon}_{11} \geq 10^{4}$. Fig. 11 presents the $\bar{\sigma}_{11} \sim \varepsilon_{11}$ curves with the conversion of $\bar{\sigma}_{11}=\sigma_{11}+p$. The symbols represent the necking points of the stress strain curves. As expected, Figs. 10 and 11 clearly show that the superimposed hydrostatic pressure 1) does not affect work-hardening, 2) lowers the $\sigma_{11} \sim \varepsilon_{11}$ curve vertically by the amount of $p$ and, 3) increases the limit strain.

The effect of superimposed hydrostatic pressure on the predicted major limit strains under three strain paths is shown in Fig. 12. The three strain paths are uniaxial stretching $(\rho=-0.5)$, plane strain tension $(\rho=0)$ and equi-biaxial tension $(\rho=1)$, respectively. The limit strains increase with increasing the superimposed hydrostatic pressure for all three strain paths. However the effect of superimposed hydrostatic pressure on the limit strains is strain path dependent. The amount of the limit strain increments in all three strain paths is slightly different 
from each other. Careful observation shows that the limit strain increment in equi-biaxial tension $(\rho=1)$ is larger than that in uniaxial stretching $(\rho=-0.5)$, which is larger than that in plane strain tension $(\rho=0)$.

Figure 13 presents the predicted FLDs under various superimposed pressure. The superimposed hydrostatic pressure increases sheet metal limit strain for any strain paths. The predicted major limit strain $\varepsilon_{11}^{*}$ decreases with $\rho$ to reach its lowest point at $\rho \approx 1$ for all pressures, and then increases until $\rho=0.2$. With further increasing $\rho, \varepsilon_{11}^{*}$ once again decreases. The "hump" in the right hand side of the predicted FLDs was also found by Chino et al. (2007) from the measured FLDs for magnesium alloy AZ31 sheet at elevated temperature. With increasing the superimposed hydrostatic pressure, the "hump" increases significantly. In the simulations we scanned every $5^{\circ}$ of a range of initial groove orientation $\psi_{I}$ and then determined the critical groove angle that gives the minimum limit strain. Fig. 14 gives the predicted critical groove orientation. It is observed that, for all the superimposed hydrostatic pressure $p$, a groove oriented at $\psi_{I}=0^{\circ}$ is favorable for necking when $-0.3 \leq \rho \leq 0.8$ except for $\rho=0.2$ at $p=200 \mathrm{MPa}$, where the critical groove orientation is $\psi_{I}=10^{\circ}$. At uniaxial tension $(\rho=-0.5)$ the critical groove orientations are $\psi_{I}=0^{\circ}$ for $p=0 \mathrm{MPa}, \psi_{I}=5^{\circ}$ for $p=40,80 \mathrm{MPa}$ and $\psi_{I}=10^{\circ}$ for $p=120,160,200 \mathrm{MPa}$. At $\rho=-0.4$ the critical groove orientation $\psi_{I}=0^{\circ}$ for $p=0,40,80$, $120 \mathrm{MPa}, \psi_{I}=5^{\circ}$ for $p=160 \mathrm{MPa}$ and $\psi_{I}=10^{\circ}$ for $p=200 \mathrm{MPa}$ are calculated. At $\rho=0.9$ the critical orientation $\psi_{I}=35^{\circ}$ is calculated for $p=40 \mathrm{MPa}$, while $\psi_{I}=0^{\circ}$ is found for all other superimposed hydrostatic pressures. At equi-biaxial tension $(\rho=1)$ the critical value $\psi_{I}=45^{\circ}$ is found for all superimposed hydrostatic pressures. 
Figure 15 presents the relative activity of the plastic deformation mechanism outside the groove under plane tension with various superimposed hydrostatic pressures. The activity of the deformation mechanisms is similar to each other at different superimposed hydrostatic pressure; except that the necking strain increases with increasing the pressure. Therefore, the hydrostatic pressure only involves the elastic deformation and does not change the plastic deformation activities. Fig. 16 shows the non-uniform inhomogeneities $f$ at the end of the two deformation processes of superimposing the hydrostatic pressure and plane strain tension (necking point at $\rho=0$ ). The non-uniform inhomogeneity $f$ keeps almost constant $(f=0.99)$ after superimposing the hydrostatic pressure. However, the non-uniform inhomogeneity $f$ at necking point decreases significantly with increasing the hydrostatic pressure $p$. It indicates that hydrostatic pressure prevents the magnesium alloy from necking even the non-uniform inhomogeneity is evolved to be very significant. This finding is consistent with the experimental observations on steel by Kao et al. (1990) and on aluminum alloy Yang et al. (1992). Kao et al. found that the necking of the 1045 spheroidized steel was significantly delayed by the superimposed hydrostatic pressure. Yang et al. found that formability of aluminum alloy AA7475 can be improved by imposing hydrostatic back pressures.

In addition, Banabic and Soare (2008) showed that the effect of the normal stress $\sigma_{33}$ on FLDs was similar to that of hydrostatic pressure. The effect of the normal stress $\sigma_{33}$ on FLDs of AZ31B sheet is studied and shown in Fig. 17. The predicted FLDs are indeed very similar to those under the superimposed hydrostatic pressure. Fig. 18 demonstrates the effects of superimposed hydrostatic pressure and normal stress on the major limit strain for plane strain tension $(\rho=0)$. The superimposed pressure and normal stress have the same effect on the limit 
strains up to the value of $320 \mathrm{MPa}$. However, it is important to distinguish the effects of the hydrostatic pressure $\sigma_{11}=\sigma_{22}=\sigma_{33}=-p$ and the normal stress component $\sigma_{33}$ on FLDs. Although the stress condition is initialized to a fixed hydrostatic pressure, the in-plane components of this pre-stress are rapidly erased when a subsequent straining is imposed, and all left is the normal component $\sigma_{33}$. However, $\mathrm{Wu}$ et al. (2009) have pointed out that this observation depends on the magnitude of the superimposed hydrostatic pressure $p$ and the subsequent strain path $\rho$. The difference in the predicted FLDs of the magnesium alloy sheets between these two pre-stress states may be noticeable when magnitude of the stress is large enough. Here we did not investigate this difference because very high hydrostatic pressure could cause very large in-plane stretching and necking should not be the failure mechanism, which is out of the scope of current study.

The use of the Jaumann rate in Eqn. (1) may influence the numerical results to some extent. However, we do not think it could change the general conclusions made in the present paper. It is noted that Jaumann rate in Eqn. (1) is with respect to the lattice spin tensor $\boldsymbol{w}^{e}$. Wu et al. (2009) applied a classical phenomenological plasticity model, which uses Jaumann rate with respect to the continue spin tensor $\boldsymbol{w}$. It is also noted that Smith et al. (2003) and Allwood and Shouler (2009) discussed the effect of normal stress (perpendicular to the sheet plane) on FLDs. In their simulations, an objective stress rate was not used at all. However, they also concluded that the normal stress increases FLDs, which is consistent with the results shown in Fig. 17 in the present paper. The above observations indicate that whether or not using an objective stress rate does not influence the conclusion on the effect of superimposed hydrostatic pressure on FLDs.

\section{Conclusions}


In this paper, we have calculated FLDs based on the recently developed elastic-viscoplastic self-consistent (EVPSC) model, in conjunction with the M-K approach, for magnesium alloy AZ31B sheet. The influence of differential speed rolling, superimposed hydrostatic pressure and elasticity on formability of magnesium alloy sheet was systematically investigated. The following conclusions can be drawn:

1) The elastic constants have noticeable effect on the predicted FLDs. Higher FLDs are predicted with higher elastic constants. Anisotropic elastic constants also give higher predicted FLDs than the isotropic ones.

2) Formability of AZ31B sheet can be improved by imposing shear deformation (e.g. DSR) to weaken the basal texture without grain refinement. The optimized improvement of the formability is obtained at shear deformation of $\gamma \approx 0.3$.

3) Formability of AZ31B sheet can be improved by imposing hydrostatic pressure, which significantly delays the onset of necking of the magnesium alloy sheets. The larger the imposed hydrostatic pressure is, the higher the predicted FLDs are.

\section{Acknowledgement}

This research was supported by funding from the NSERC Magnesium Strategic Research Network. More information on the Network can be found at www.MagNET.ubc.ca. HW and JW were supported by the US department of Energy, Office of Basic Energy Sciences (Project No: FWP-06SCPE401).

\section{References}

Agnew S.R., Horton J.A., Lillo T.M. and Brown D.W., 2004. Enhanced ductility in strongly textured magnesium produced by equal channel angular processing. Scripta Materialia 50, 377-381.

Allwood J.M., Shouler D.R., 2009. Generalised forming limit diagrams showing increased forming limits with non-planar stress states. International Journal of Plasticity 25, 12071230.

Asaro R.J. and Needleman A., 1985. Texture development and strain hardening in rate dependent polycrystals. Acta Metallurgica Et Materialia 33, 923-953. 
Ashby M.F., Embury J.D., Cooksley S.H. and Teirlinck, D., 1985. Fracture maps with pressure as a variable. Scripta Metallurgica 19, 385-390.

Banabic D. and Soare S., 2008. On the effect of the normal pressure upon the forming limit strains. In: Hora, P. (Ed.), Numisheet 2008 - Proceedings of the 7th International Conference and Workshop on Numerical Simulation of 3D Sheet Metal Forming Processes. pp. 199-204.

Bridgman P.W., 1952. Studies in large plastic flow and fracture - with special emphasis on the effects of hydrostatic pressure. McGraw-Hill, New York.

Brownrigg A., Spitzig W.A., Richmond O., Teirlinck D. and Embury, J.D., 1983. The influence of hydrostatic-pressure on the flow-stress and ductility of a spherodized 1045-steel. Acta Metallurgica 31, 1141-1150.

Chino Y., Iwasaki H., and Mabuchi M., 2007. Stretch formability of AZ31 Mg alloy sheets at different testing temperatures. Materials Science and Engineering A466, 90-95.

Chino Y., Sassa K. and Mabuchi M., 2008. Enhancement of tensile ductility of magnesium alloy produced by torsion extrusion. Scripta Materialia 59, 399-402.

Eshelby J.D., 1957. The determination of the elastic field of an ellipsoidal inclusion, and related problems. Proceedings of the Royal Society of London A241, 376-396.

French L.E. and Weinrich P.F., 1975. The influence of hydrostatic pressure on the tensile deformation and fracture of copper. Metallurgical Transactions 6A, 785-790.

French L.E., Weinrich P.F. and Weaver C.W., 1973. Tensile fracture of free machining brass as a function of hydrostatic pressure. Acta Metallurgica 21, 1045-1049.

Gimple J.L., Wilkinson D.S., Embury J.D. and Lewandowski, J.J., 2001. Effect of superimposed pressure on the fracture behavior of aluminum automotive alloys. In: Das, S.K., Kaufman, J.G., Lienert, T.J. (Eds.), Aluminum 2001-Proceedings of the TMS 2001, TMS,17-29.

Gupta M. and Sharon N.M.L., 2011. Magnesium, Magnesium Alloys, and Magnesium Composites. John Wiley.

Hecker S.S., 1975. Formability of aluminum alloy sheet. Journal of Engineering Materials and Technology 97, 66-73.

Huang X.S., Suzuki K., Watazu A., Shigematsu I. and Saito N., 2008. Mechanical properties of Mg-Al-Zn alloy with a tilted basal texture obtained by differential speed rolling. Materials Science and Engineering A488, 214-220.

Jain A. and Agnew S.R., 2007. Modeling the temperature dependent effect of twinning on the behavior of magnesium alloy AZ31B sheet. Materials Science and Engineering A462, 29-36.

Kao A.S., Kuhn H.A., Richmond O. and Spitzig W.A., 1989. Workability of 1045 spheroidized steel under superimposed hydrostatic-pressure. Metallurgical Transactions 20A, 17351741. 
Kao A.S., Kuhn H.A., Spitzig W.A. and Richmond O., 1990. Influence of superimposed hydrostatic-pressure on bending fracture and formability of a low-Carbon steel containing globular sulfides. Journal of Engineering Materials and Technology 112, 26-30.

Korbel A., Raghunathan V.S., Teirlinck D., Spitzig W., Richmond O. and Embury J.D., 1984. A structural study of the influence of pressure on shear band formation. Acta Metallurgica 32, 511-519.

Kurukuri S., Worswick M.J., Ghaffari Tari D., Mishra R.K., Carter J.T., 2014. Rate sensitivity and tension-compression asymmetry in AZ31B magnesium alloy sheet. Philosophical Transactions of the Royal Society A372: 20130216.

Lee S.Y., Wang H., Gharghouri M.A., Nayyeri G., Woo W., Shin E., Wu P.D., Poole W.J., Wu W. and An K., 2014. Deformation behavior of solid-solution-strengthened Mg-9 wt.\% Al alloy: In situ neutron diffraction and elastic-viscoplastic self-consistent modeling. Acta Materialia 73, 139-148.

Lewandowski J.J. and Lowhaphandu P., 1998. Effects of hydrostatic pressure on mechanical behaviour and deformation processing of materials. International Materials Reviews 43, 145-187.

Liu D.S. and Lewandowski J.J., 1993. The effects of superimposed hydrostatic-pressure on deformation and fracture 2. particulate-reinforced-6061 composites. Metallurgical Transactions 24A, 609-615.

Marciniak Z. and Kuczynski K., 1967. Limit strains in the process of stretch-forming sheet metal. International Journal of Mechanical Sciences 9, 609-620.

Mukai T., Yamanoi M., Watanabe H. and Higashi K., 2001. Ductility enhancement in AZ31 magnesium alloy by controlling its grain structure. Scripta Materialia 45, 89-94.

Neil J.C. and Agnew S.R., 2009. Crystal plasticity-based forming limit prediction for non-cubic metals: application to Mg alloy AZ31B. International Journal of Plasticity 25, 379-398.

Ratchev P., Van Houtte P., Verlinden B., De Smet P., Neutjens P., Baartman R., Drent P., 1994. Prediction of forming limit diagrams of Al-Mg rolled sheet taking texture. Textures and Microstructures 22, 219-231.

Simmons G. and Wang H., 1971. Single crystal elastic constants and calculated polycrystal properties. Cambridge (MA): MIT Press.137.

Smith L.M., Ganeshmurthy S., Alladi K., (2003) Double-sided high-pressure tubular hydroforming. Journal of Materials Processing Technology, 142, 599-608.

Spitzig W.A. and Richmond O., 1984. The effect of pressure on the flow-stress of metals. Acta Metallurgica 32, 457-463.

Suzuki K., Chino Y., Huang X. and Mabuchi M. 2011. Elastic and damping properties of AZ31 magnesium alloy sheet processed by high-temperature rolling. Materials Transactions 52, 2040-2044. 
Tomé C.N., Lebensohn R.A. and Kocks U.F., 1991. A model for texture development dominated by deformation twinning - application to zirconium alloys. Acta Metallurgica Et Materialia 39, 2667-2680.

Wang H., Clausen B., Tomé C.N., Wu P.D., 2013a. Studying the effect of stress relaxation and creep on lattice strain evolution of stainless steel under tension. Acta Materialia 61, 11791188.

Wang H., Raeisinia B., Wu P.D., Agnew S.R. and Tomé C.N., 2010a. Evaluation of selfconsistent crystal plasticity models for magnesium alloy AZ31B sheet. International Journal of Solids and Structures 47, 2905-2917.

Wang H., Wu P.D., Boyle K.P. and Neale K.W., 2011. On crystal plasticity formability analysis for magnesium alloy sheets. International Journal of Solids and Structures 48, 1000-1010.

Wang H., Wu P.D. and Neale K.W., 2010b. On the role of the constitutive model and basal texture on the mechanical behavior of magnesium alloy AZ31B sheet. Journal of Zhejiang University-SCIENCE A11, 744-755.

Wang H., Wu P.D. and Gharghouri M.A., 2010c. Effects of basal texture on mechanical behaviour of magnesium alloy AZ31B sheet. Materials Science and Engineering A527, 3588-3594.

Wang H., Wu P.D., Tomé C.N. and Huang Y., 2010d. A finite strain elastic-viscoplastic selfconsistent model for polycrystalline materials. Journal of the Mechanics and Physics of Solids 58, 594-612.

Wang H., Wu Y., Wu P.D. and Neale K.W., 2010e. Numerical analysis of large strain simple shear and fixed-end torsion of HCP polycrystals. CMC-COMPUTERS MATERIALS \& CONTINUA, 19, 255-284.

Wang H., Wu P.D., Tomé C.N. and Wang J. 2012a. Study of lattice strains in magnesium alloy AZ31 based on a large strain elastic-viscoplastic self-consistent polycrystal model. International Journal of Solids and Structures 49, 2155-2167.

Wang H., Wu P.D. Tomé C.N. and Wang J., 2012b. A constitutive model of twinning and detwinning for hexagonal close packed polycrystals. Materials Science and Engineering: A555, 93-98.

Wang H., Wu P.D. and Wang J., 2013b. Modeling inelastic behavior of magnesium alloys during cyclic loading-unloading. International Journal of Plasticity 47, 49-64

Wang H., Wu P.D. Wang J. and Tomé C.N., 2013c. A crystal plasticity model for hexagonal close packed (HCP) crystals including twinning and de-twinning mechanisms. International Journal of Plasticity 49, 36-52.

Wang H., Wu P.D. and Wang J., 2015. Modelling the role of slips and twins in magnesium alloys under cyclic shear. Computational Materials Science 96, 214-218.

Wang Y.C. and Mora P., 2008. Macroscopic elastic properties of regular lattices. Journal of the Mechanics and Physics of Solids 56, 3459-3474. 
Weinrich P.F. and French I.E., 1976. Influence of hydrostatic-pressure on fracture mechanisms of sheet tensile specimens of copper and brass. Acta Metallurgica 24, 317-322.

Wu P.D., Embury J.D., Lloyd D.J., Huang Y. and Neale K.W., 2009. Effects of superimposed hydrostatic pressure on sheet metal formability. International Journal of Plasticity 25, 1711-1725.

Wu P.D., Neale K.W. and Van der Giessen E., 1997. On crystal plasticity FLD analysis. Proceedings of the Royal Society A453, 1831-1848.

Wu P.D., Neale K.W., Van der Giessen E., Jain M., Makinde A. and MacEwen S.R., 1998. Crystal plasticity forming limit diagram analysis of rolled aluminum sheets. Metallurgical and Materials Transactions 29A, 527-535.

Wu P.D., Wang H. and Neale K.W., 2012. On the large strain torsion of HCP polycrystals. International Journal of Applied Mechanics 4, 1250024.

Yang H.S., Mukherjee A.K. and Roberts W.T., 1992. Effects of hydrostatic pressure and nonuniform microstructure on superplastic forming and postformed tensile properties of AA 7475 aluminium alloy. Materials Science and Technology 8, 611-619. 


\section{Table titles:}

Table 1. List of values of material constants for EVPSC model.

\section{Figure captions:}

Figure 1. The geometry and convention employed in the FLD analysis.

Figure 2. Textures of AZ31B sheet obtained by shear deformation in terms of the $\{0001\}$ and $\{10 \overline{1} 0\}$ pole figures.

Figure 3. True stress and plastic strain curves under uniaxial tension/compression along the RD. The experimental data are taken from Jain and Agnew (2007).

Figure 4. Schematic representation of the decomposition of the differential speed rolling (DSR).

Figure 5. Effect of single crystal elastic constants on the predicted FLDs.

Figure 6. The intensity of basal texture as a function of the shear deformation $\gamma$ at cut-off angles of $15^{\circ}$ and $45^{\circ}$.

Figure 7. Influence of shear deformation on the predicted FLDs.

Figure 8. Influence of shear deformation on the predicted major limit strains for strain paths of $\rho=-0.5,0$ and 1.

Figure 9. Relative activity of the deformation mechanisms outside the groove under plane tension with various shear deformed textures.

Figure 10. Plane strain tensile stress strain curves under superimposed hydrostatic pressures represented in terms of the true stress.

Figure 11. Plane strain tensile stress strain curves under superimposed hydrostatic pressures represented in terms of the measured stress.

Figure 12. Effect of superimposed hydrostatic pressure on major limit strains for strain paths of $\rho=-0.5,0$ and 1.

Figure 13. Effect of superimposed hydrostatic pressure on the predicted FLDs.

Figure 14. Predicted critical groove orientations for various superimposed hydrostatic pressures.

Figure 15. Relative activity of the deformation mechanisms under plane tension with various superimposed imposed pressures. 
Figure 16. The non-uniform inhomogeneity $\mathrm{f}$ after superimposed hydro pressure and at necking under plane tension $(\rho=0)$.

Figure 17. Effect of normal stress $\sigma_{33}$ on the predicted FLDs.

Figure 18. Effect of superimposed pressure and normal stress on major limit strain for plane strain tension $(\rho=0)$. 
Table 1. List of values of material constants for EVPSC model.

\begin{tabular}{cccccccc}
\hline Mode & $\boldsymbol{\tau}_{\mathbf{0}}$ & $\boldsymbol{\tau}_{\mathbf{1}}$ & $\boldsymbol{h}_{\mathbf{0}}$ & $\boldsymbol{h}_{\mathbf{1}}$ & Latent & $\boldsymbol{A}^{\text {th } 1}$ & $\boldsymbol{A}^{\text {th2 }}$ \\
\hline Basal & 9 & 1 & 50 & 25 & 4 & & \\
Prismatic & 79 & 40 & 590 & 50 & 4 & & \\
Pyramidal & 100 & 100 & 5000 & 0 & 2 & & \\
Extension Twin & 47 & 0 & 0 & 0 & 4 & 0.72 & 0 \\
\hline
\end{tabular}

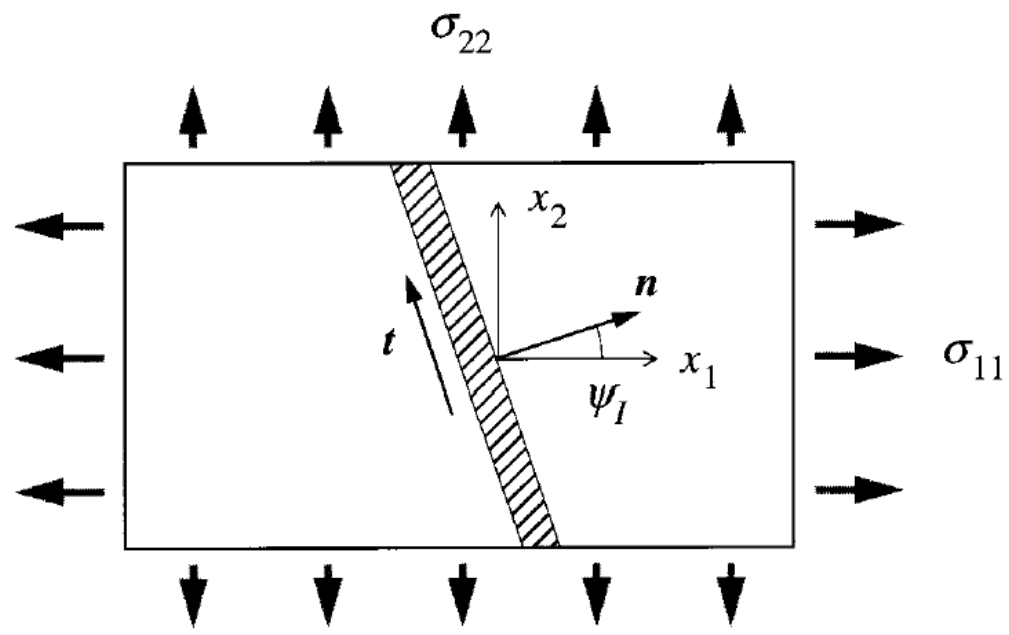

Figure 1. The geometry and convention employed in the FLD analysis.
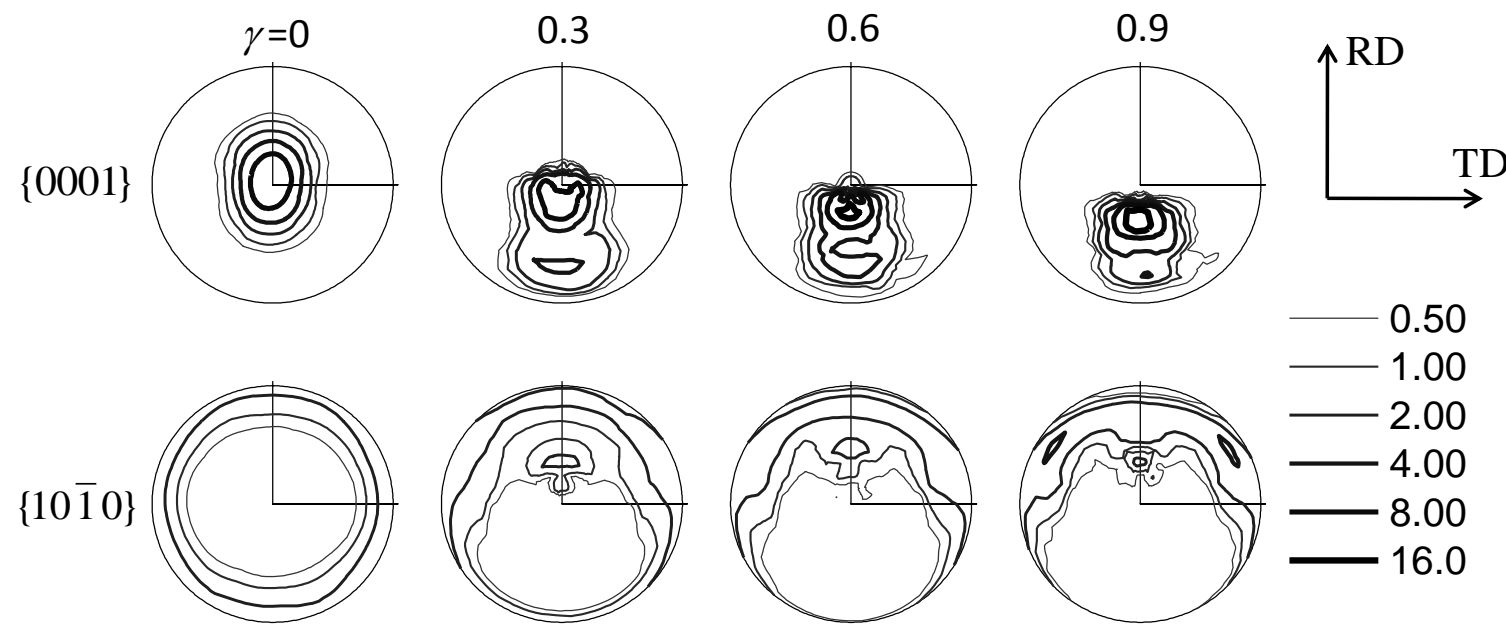

Figure 2. Textures of AZ31B sheet obtained by shear deformation in terms of the $\{0001\}$ and $\{10 \overline{1} 0\}$ pole figures. 


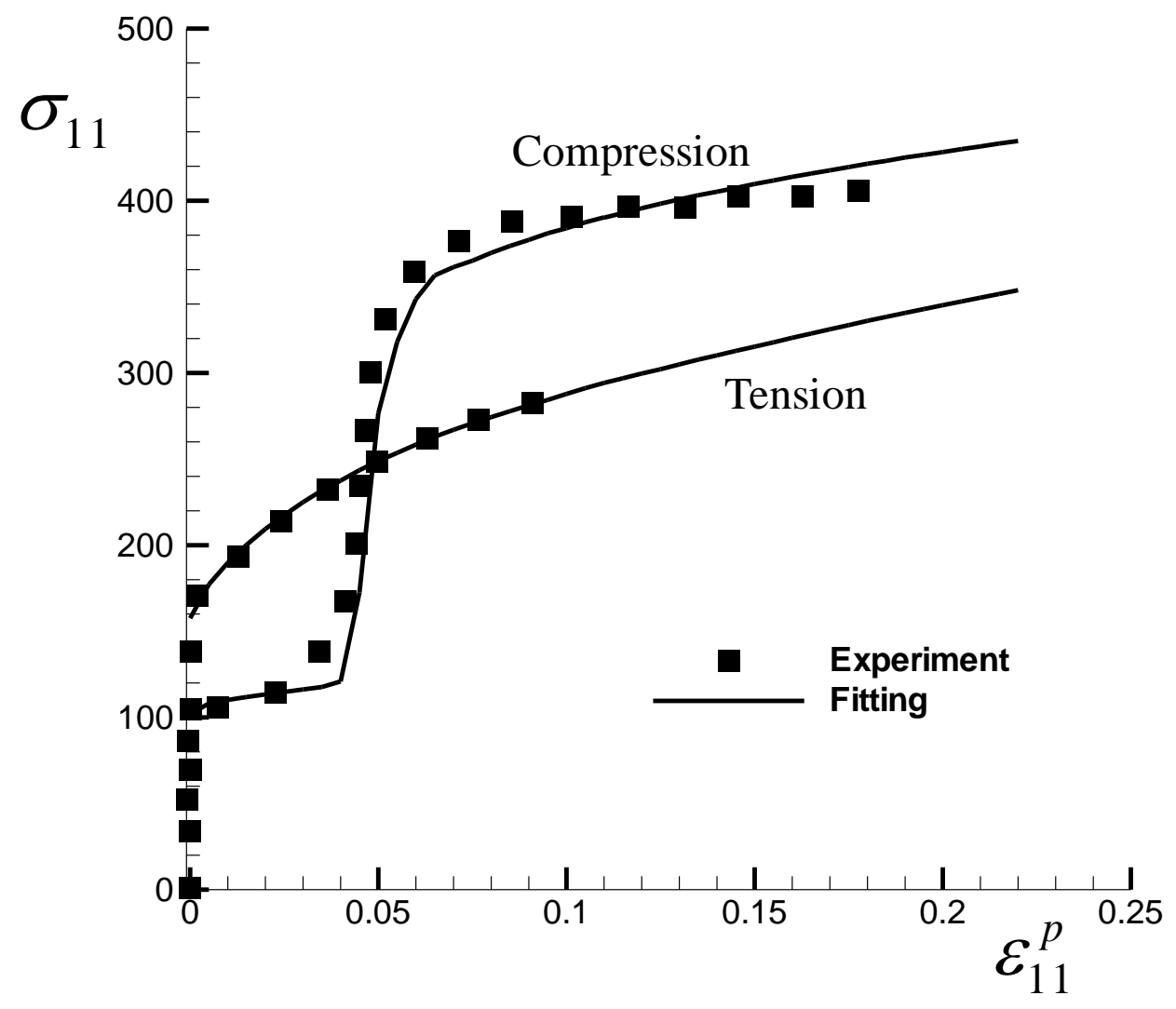

Figure 3. True stress and plastic strain curves under uniaxial tension/compression along the RD. The experimental data are taken from Jain and Agnew (2007). 


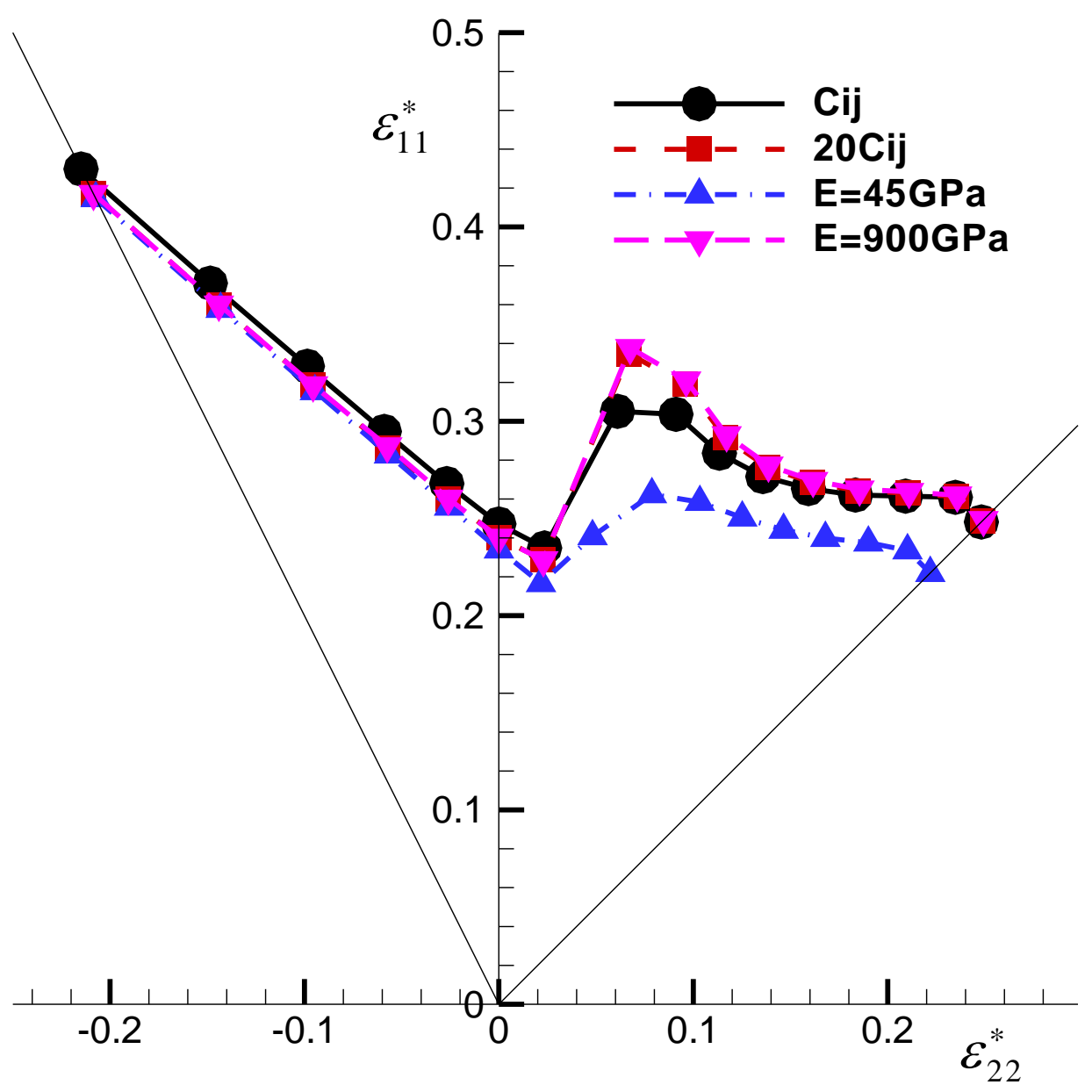

Figure 4. Effect of single crystal elastic constants on the predicted FLDs.

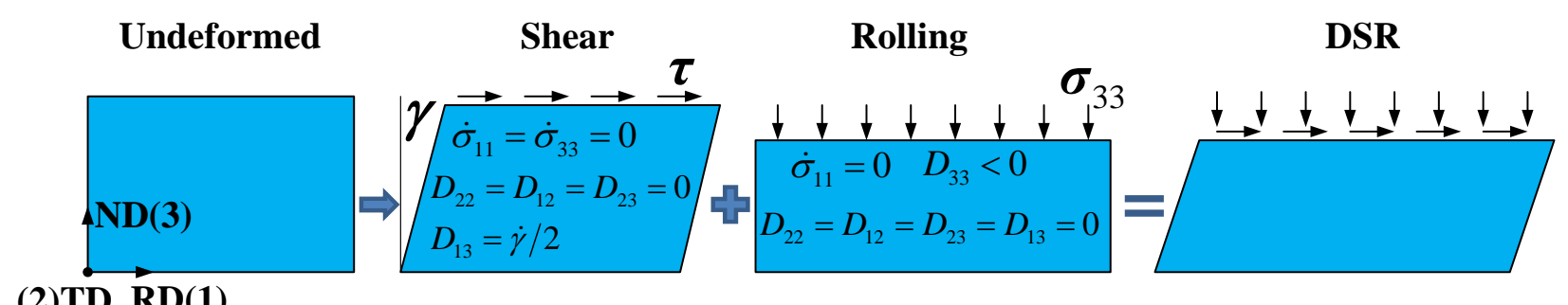

Figure 5. Schematic representation of the decomposition of the differential speed rolling (DSR). 


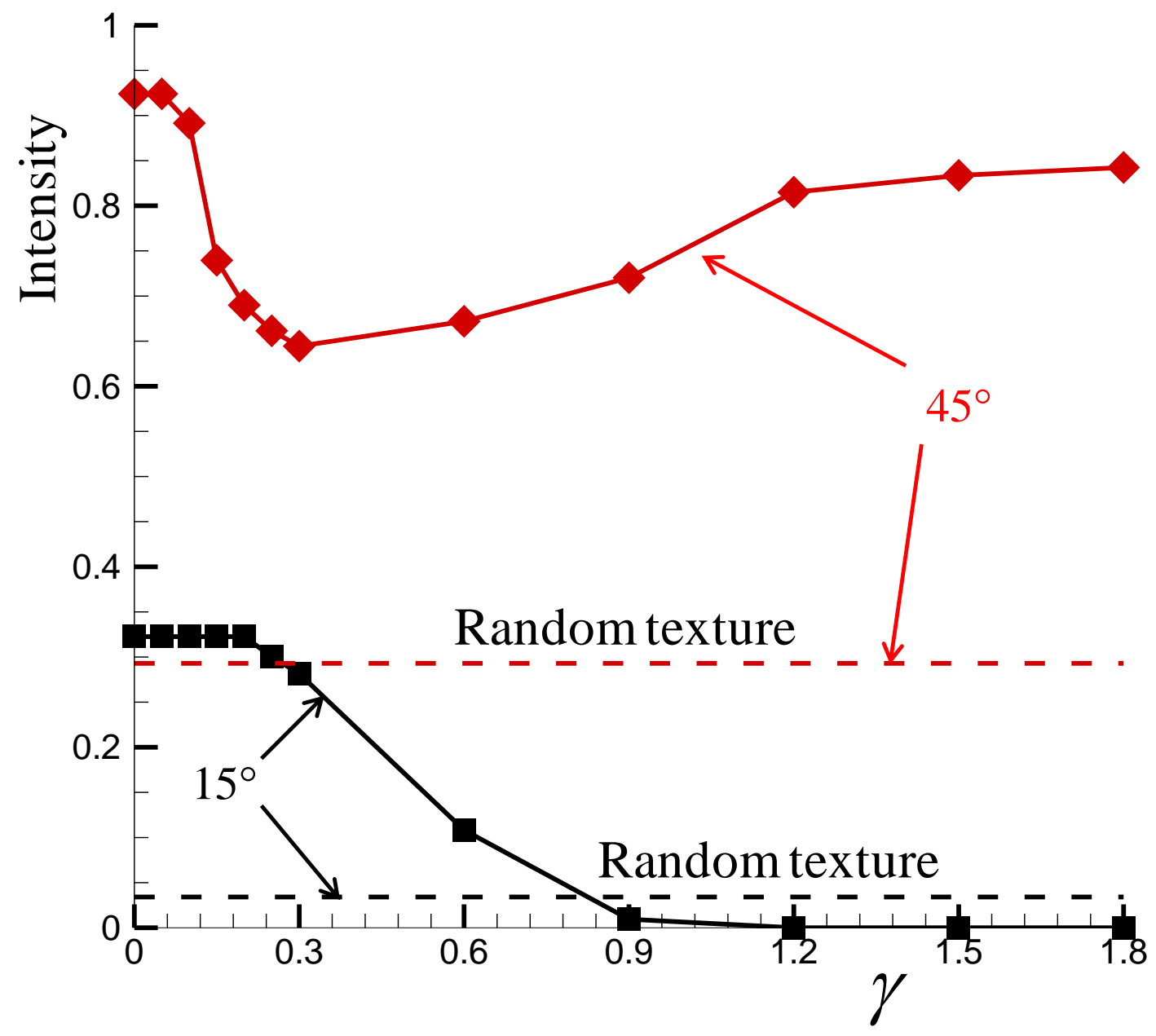

Figure 6. The intensity of basal texture as a function of the shear deformation $\gamma$ at cut-off angles of $15^{\circ}$ and $45^{\circ}$. 


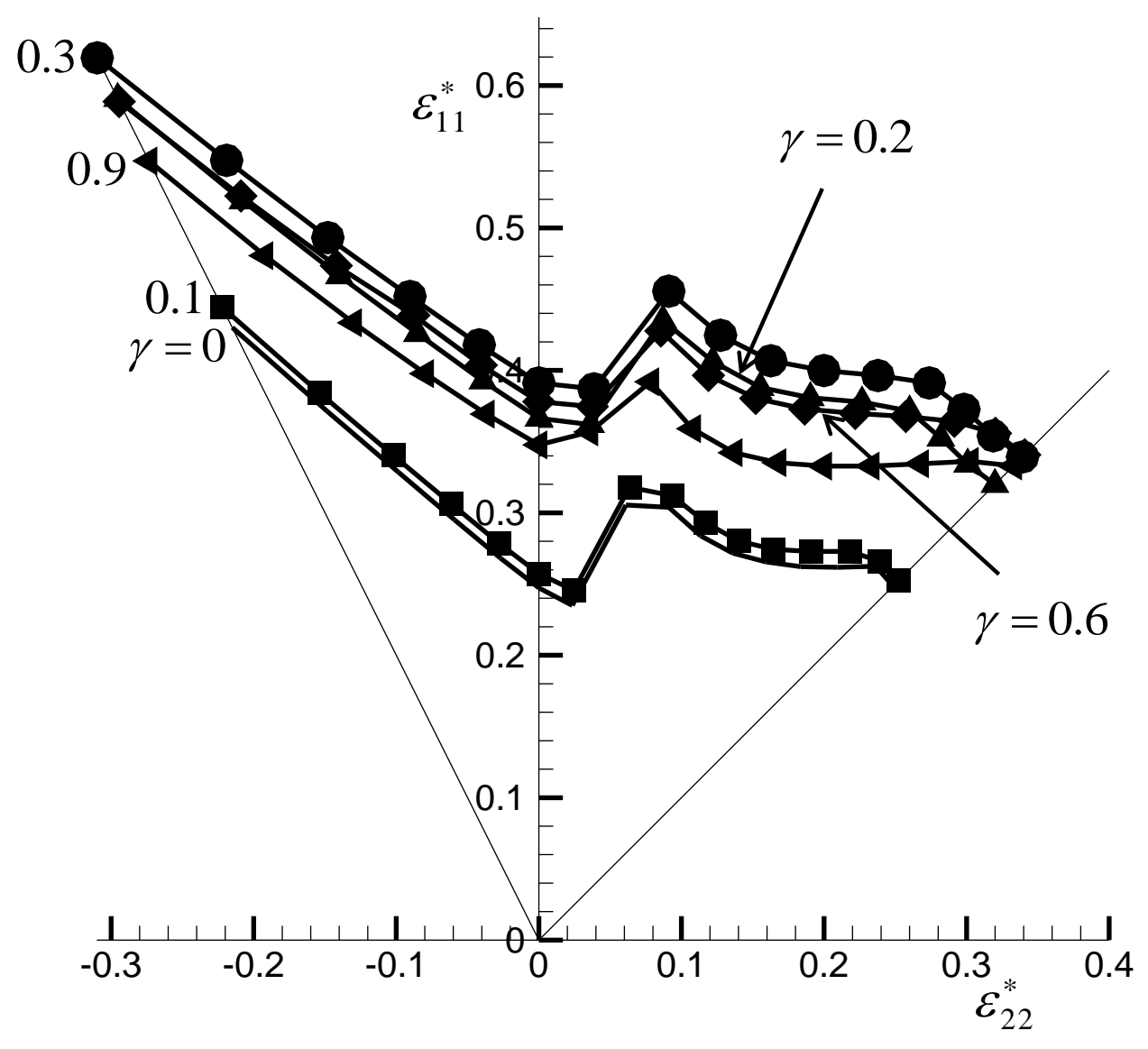

Figure 7. Influence of shear deformation on the predicted FLDs. 


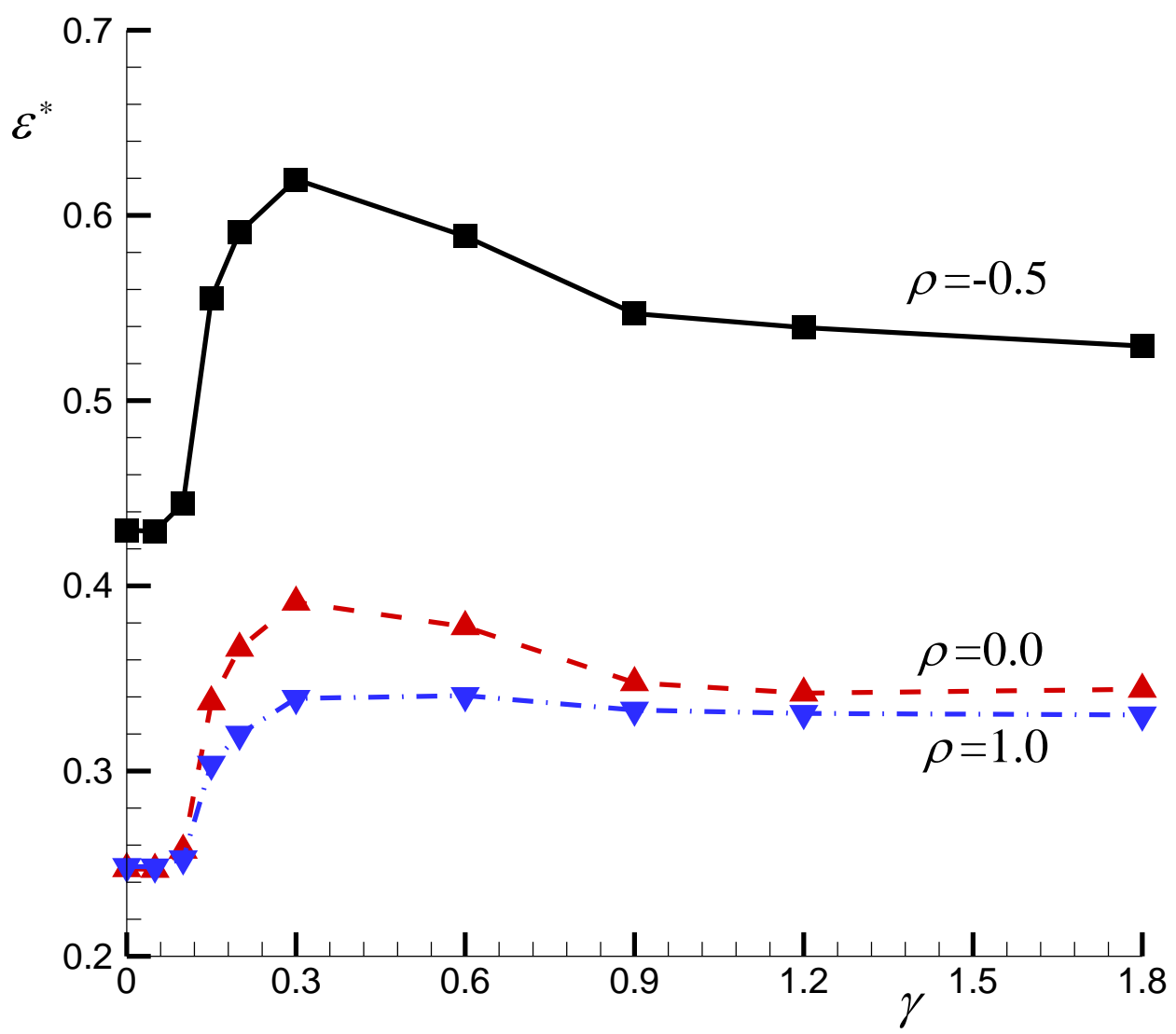

Figure 8. Influence of shear deformation on the predicted major limit strains for strain paths of $\rho=-0.5,0$ and 1 . 

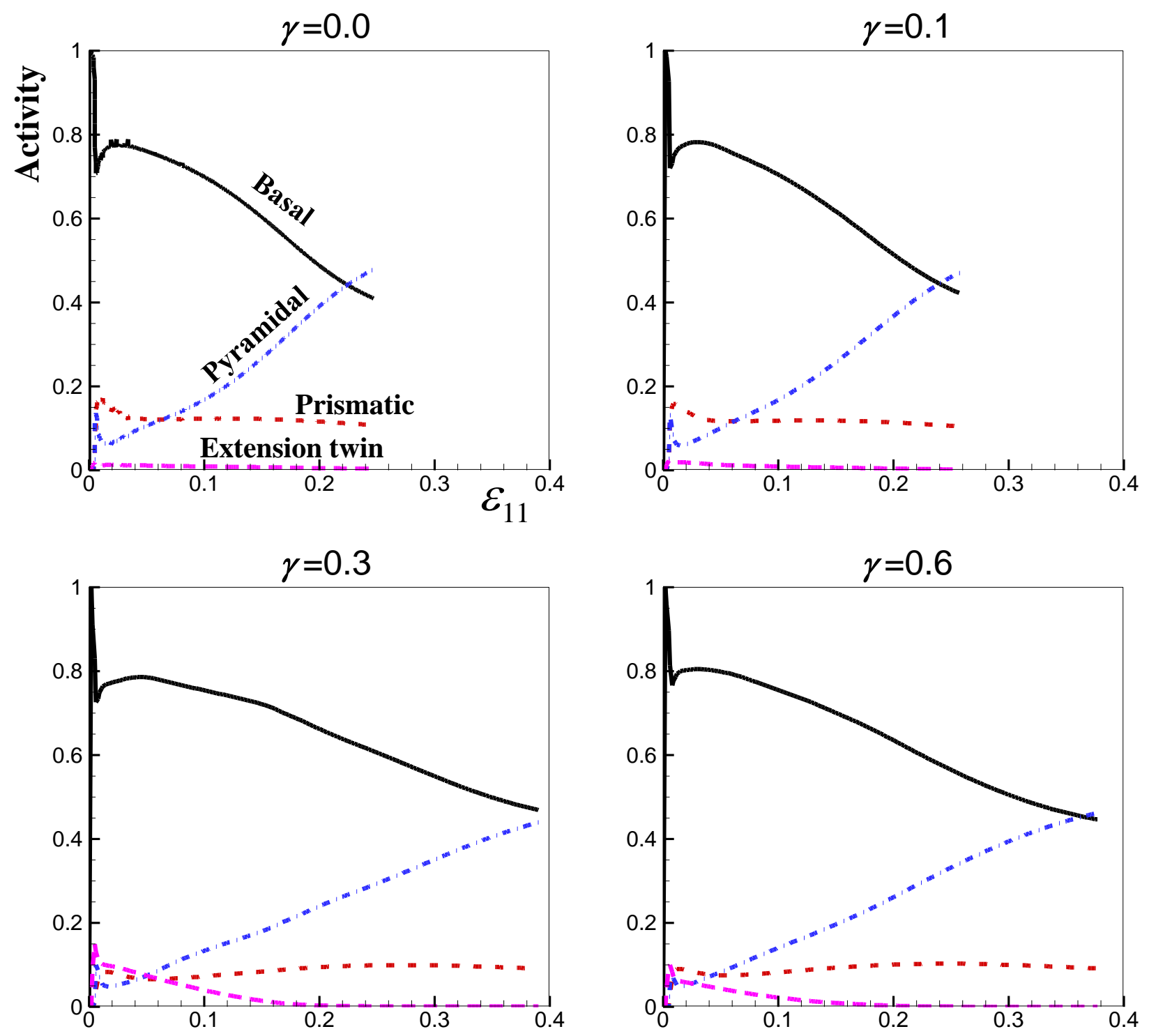

Figure 9. Relative activity of the deformation mechanisms outside the groove under plane tension with various shear deformed textures. 


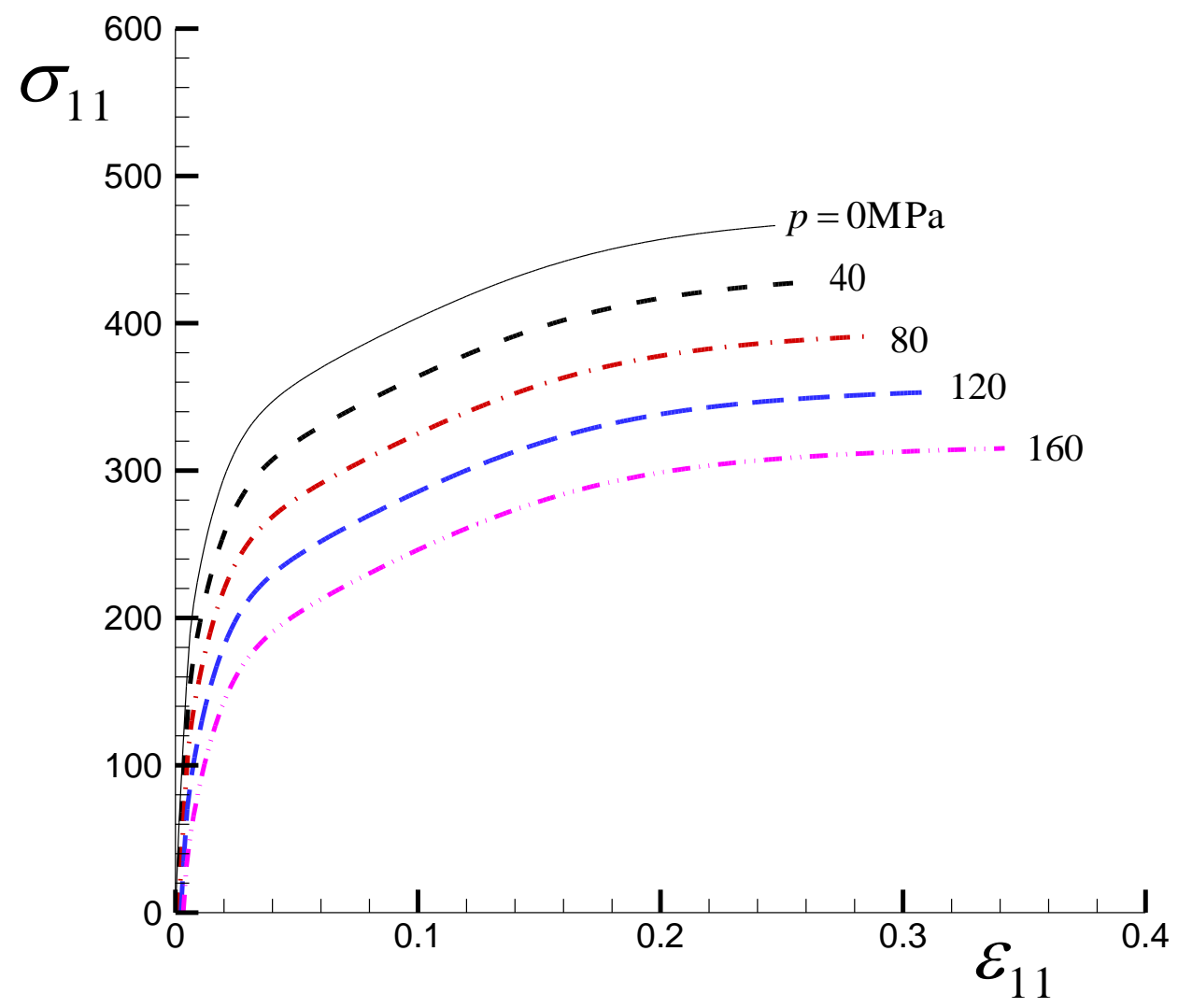

Figure 10. Plane strain tensile stress strain curves under superimposed hydrostatic pressures represented in terms of the true stress. 


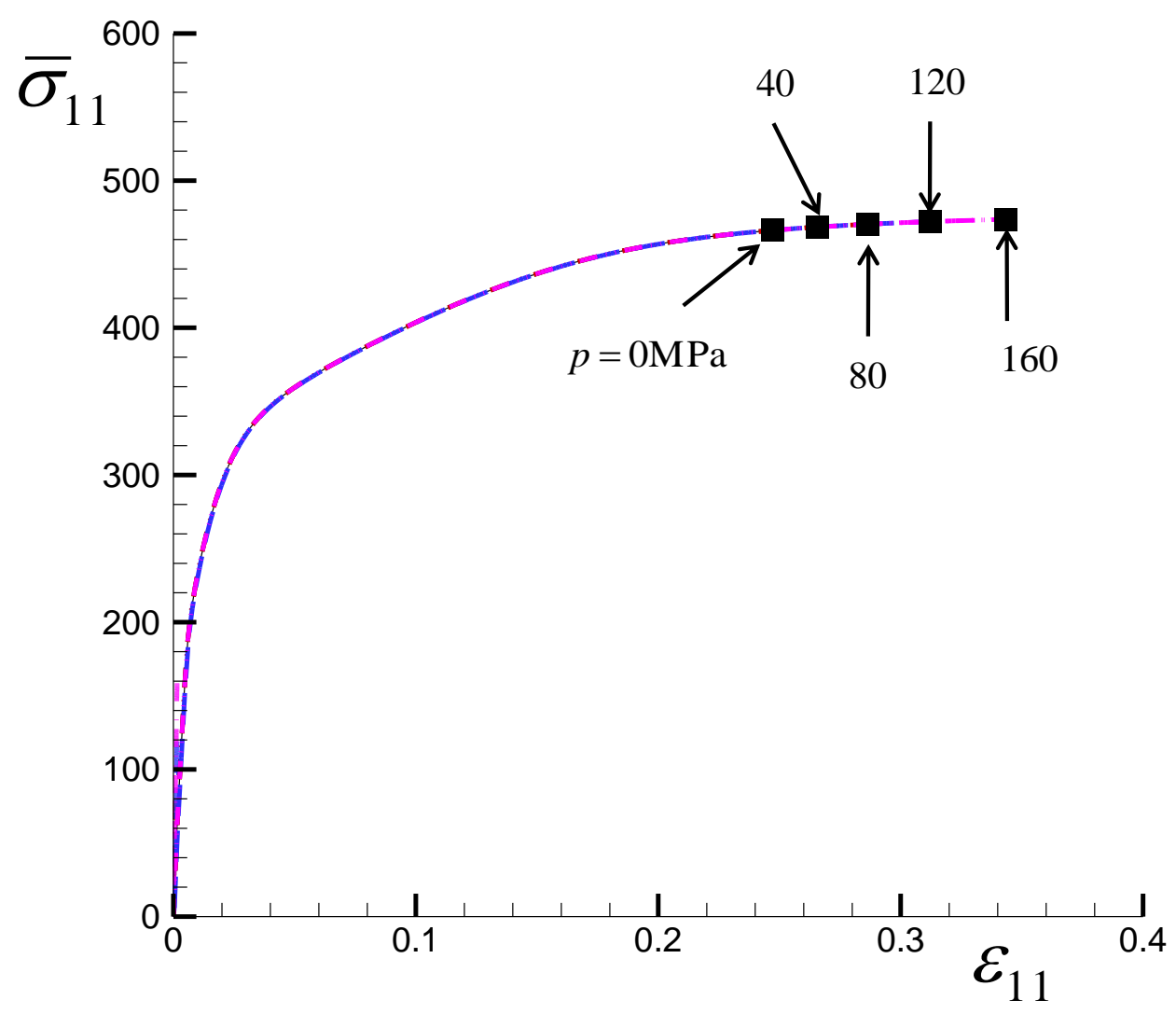

Figure 11. Plane strain tensile stress strain curves under superimposed hydrostatic pressures represented in terms of the measured stress. 


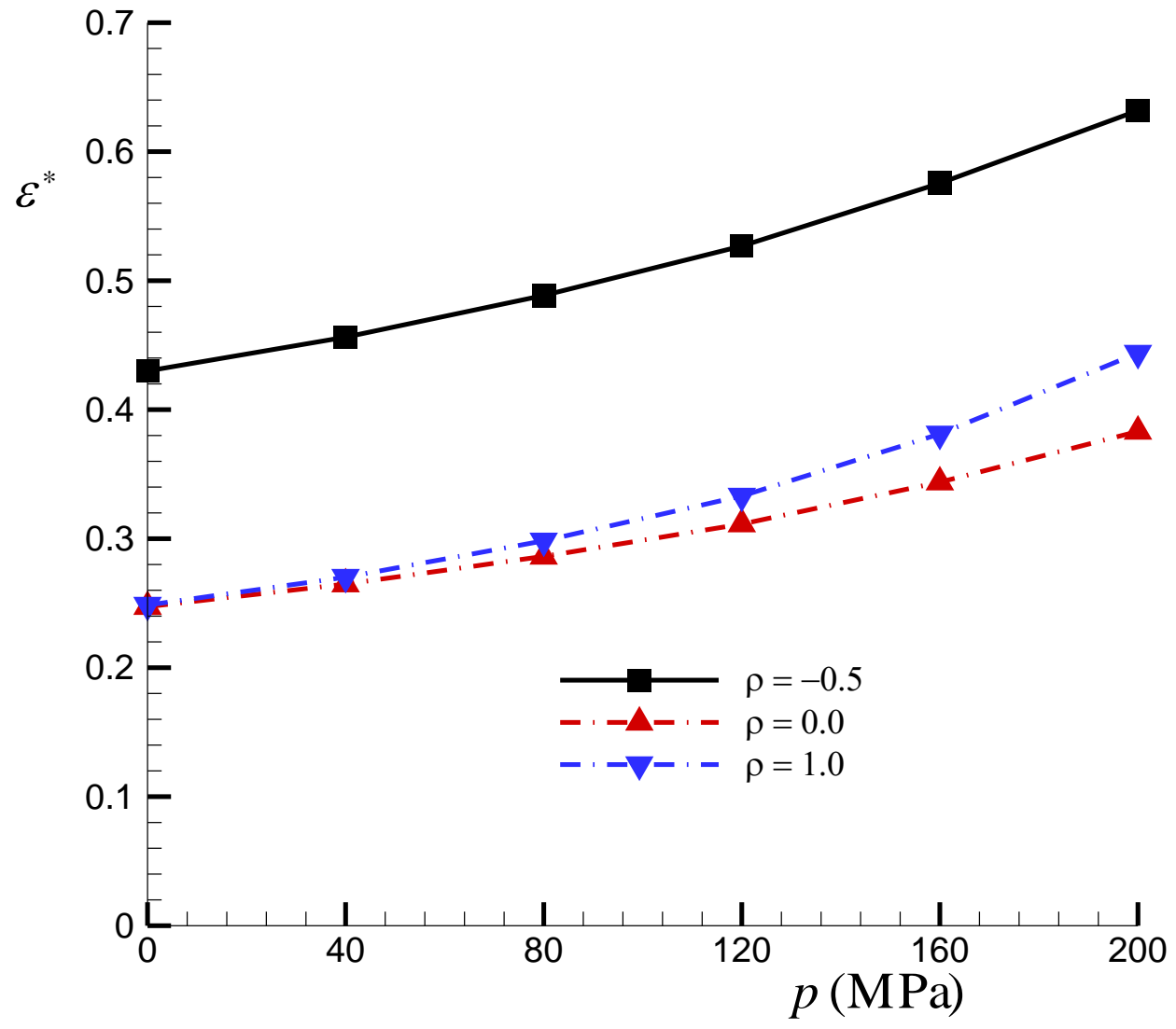

Figure 12. Effect of superimposed hydrostatic pressure on major limit strains for strain paths of $\rho=-0.5,0$ and 1 . 


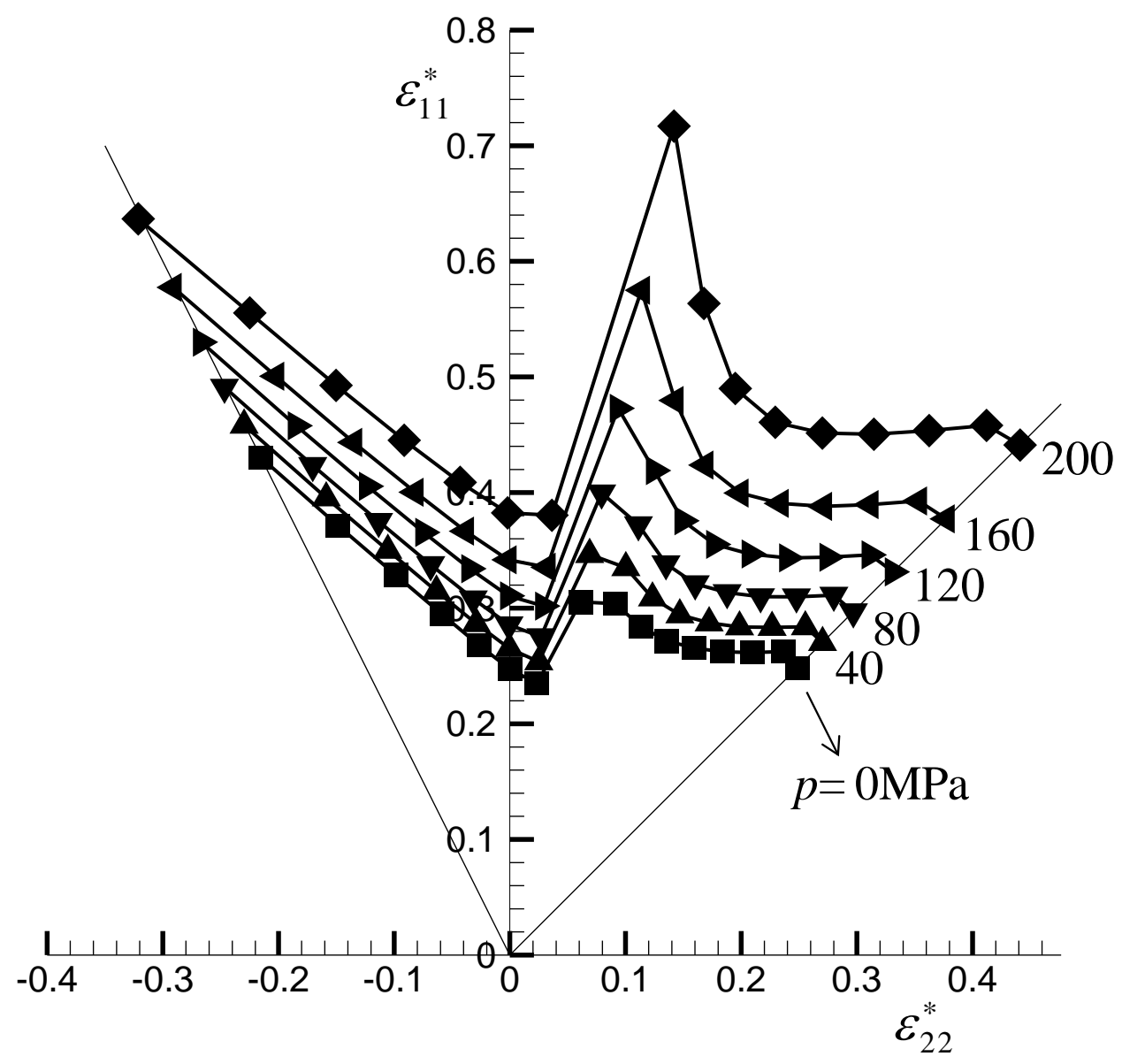

Figure 13. Effect of superimposed hydrostatic pressure on the predicted FLDs. 


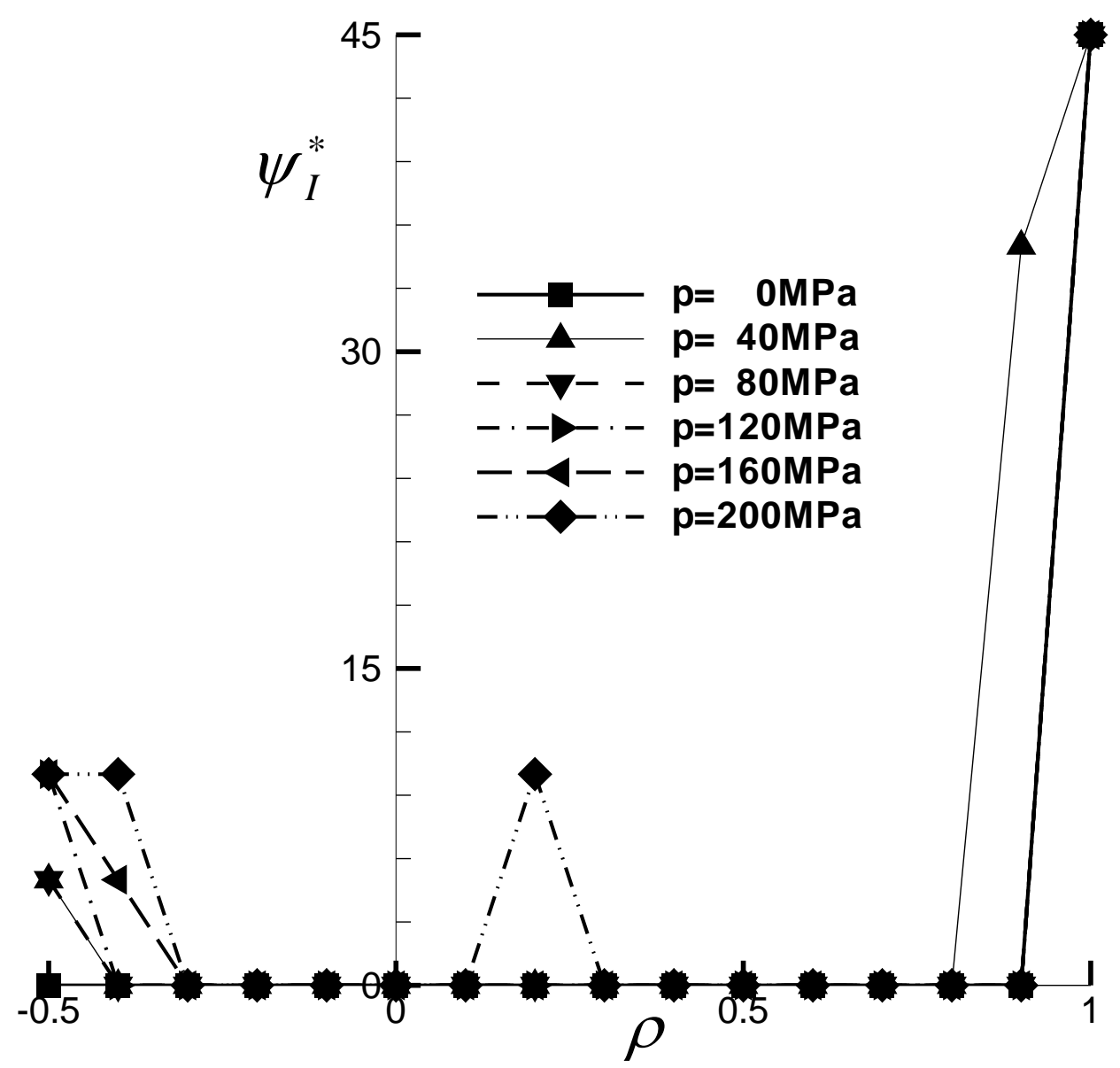

Figure 14. Predicted critical groove orientations for various superimposed hydrostatic pressures. 

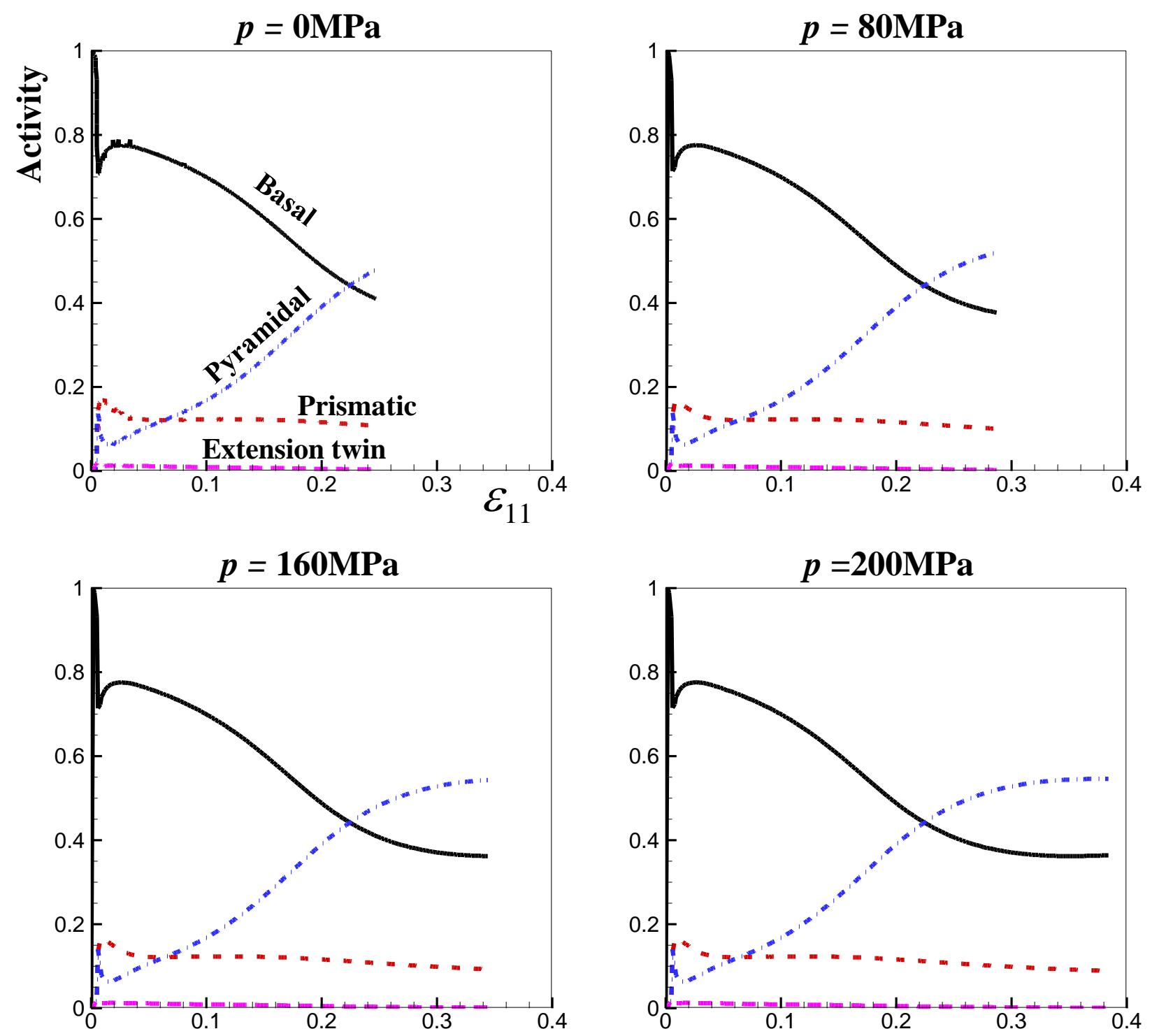

Figure 15. Relative activity of the deformation mechanisms under plane tension with various superimposed imposed pressures. 


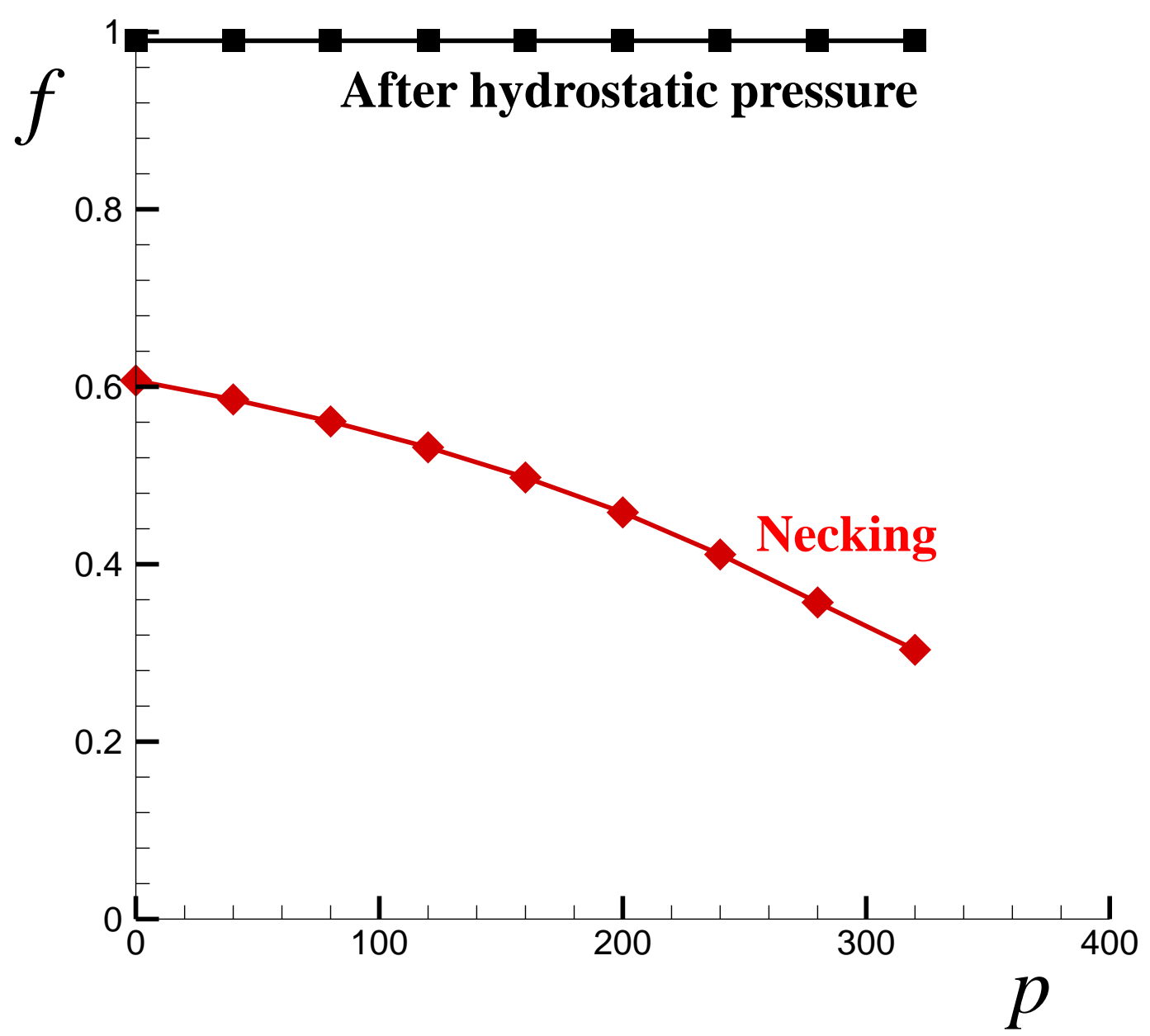

Figure 16. The non-uniform inhomogeneity $f$ after superimposed hydro pressure and at necking under plane tension $(\rho=0)$. 


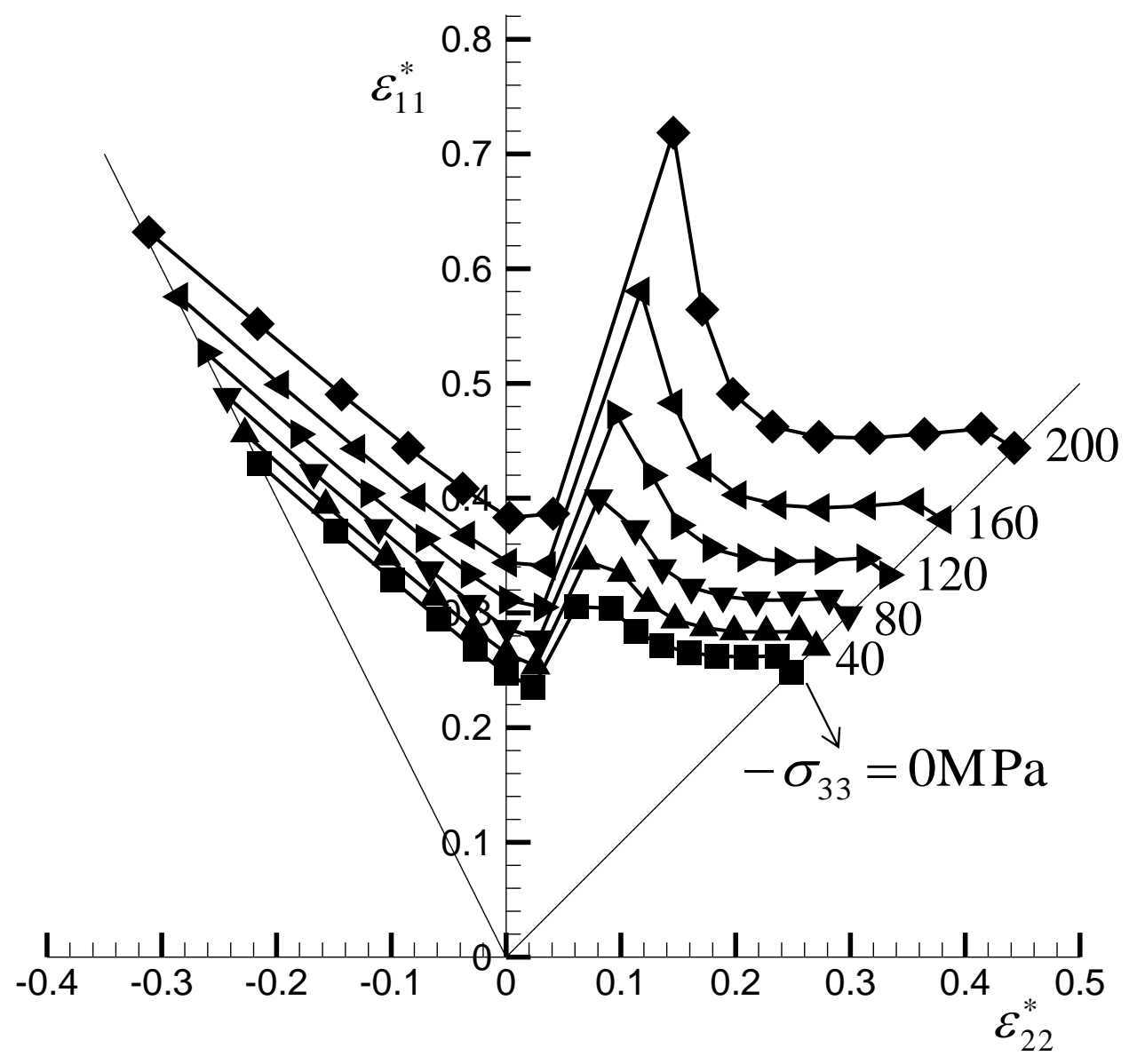

Figure 17. Effect of normal stress $\sigma_{33}$ on the predicted FLDs. 


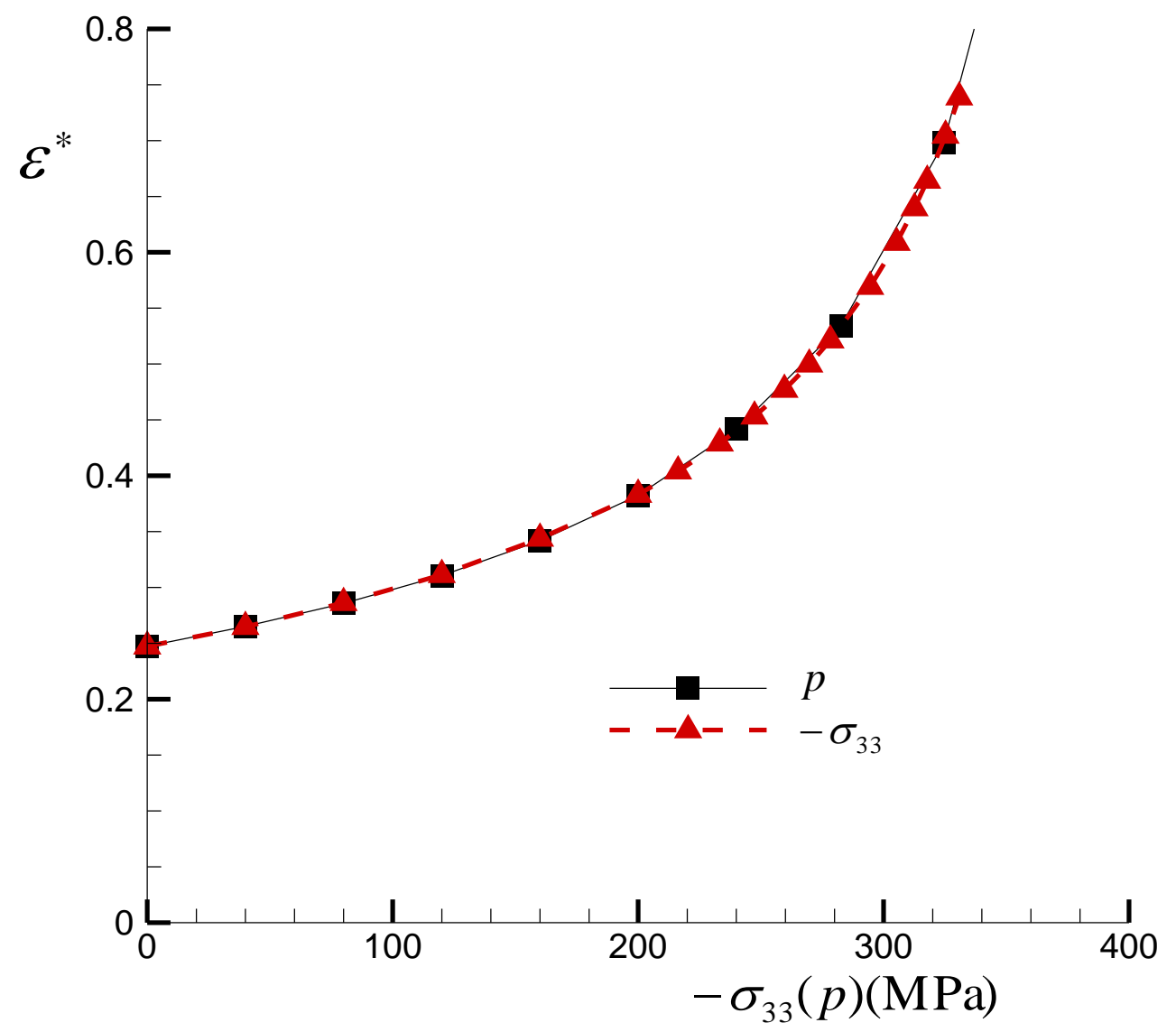

Figure 18. Effect of superimposed pressure and normal stress on major limit strain for plane strain tension $(\rho=0)$. 